\title{
WEIGHTED NORM INEQUALITIES FOR THE CONTINUOUS SQUARE FUNCTION
}

\author{
J. MICHAEL WILSON
}

This paper is dedicated to the memory of my friend Frederick J. Zeigler (1953-1988)

\begin{abstract}
We prove new weighted norm inequalities for real-variable analogues of the Lusin area function. We apply our results to obtain new: (i) weighted norm inequalities for singular integral operators; (ii) weighted Sobolev inequalities; (iii) eigenvalue estimates for degenerate Schrödinger operators.
\end{abstract}

\section{INTRODUCTION}

Let $\psi \in \mathscr{C}^{k}\left(\mathbf{R}^{d}\right)$ be real and radial, nontrivial, satisfy $\int \psi=0$, and have support contained in $\{|x| \leq 1\}$. We may clearly assume that $\psi$ is normalized so that

$$
\int_{0}^{\infty}|\hat{\psi}(\xi t)|^{2} \frac{d t}{t}=1
$$

for all $\xi \neq 0$. (Here and in the sequel, ${ }^{\wedge}$ denotes the Fourier transform.) For $y>0$ we define $\psi_{y}(x)=y^{-d} \psi\left(\frac{x}{y}\right)$. For $f \in L_{\text {loc }}^{1}\left(\mathbf{R}^{d}\right)$ and $\alpha>0$ we define

$$
S_{\psi, \alpha}(f)(x) \equiv\left(\int_{|x-t|<\alpha y}\left|f * \psi_{y}(t)\right|^{2} \frac{d t d y}{y^{d+1}}\right)^{1 / 2} .
$$

Equality (1) defines the square function of $f$ with respect to $\psi$ of aperture $\alpha$.

For $\beta=\left(\beta_{1}, \ldots, \beta_{d}\right)$ a multi-index, let $|\beta|=\sum \beta_{i}$. For $N$ a positive integer define

$$
\mathscr{A}_{n} \equiv\left\{\phi \in \mathscr{C}_{0}^{\infty}\left(\mathbf{R}^{d}\right): \operatorname{supp} \phi \subset\{|x| \leq 1\},\left\|\sum_{|\beta| \leq N}\left|D^{\beta} \phi\right|\right\|_{\infty} \leq 1\right\},
$$

Received by the editors January 18, 1988.

1980 Mathematics Subject Classification (1985 Revision). Primary 42B25, 42B20, 42B15, $81 \mathrm{C} 10$.

Key words and phrases. Lusin area function, weighted norm inequality, Calderón-Zygmund operator, Sobolev inequality, Schrödinger operator.

This research was supported by a University of Vermont Graduate Division Summer Research Fellowship. 
and for $f \in L_{\text {loc }}^{1}\left(\mathbf{R}^{d}\right)$ set

$$
G_{N}(f)(x) \equiv \sup _{\substack{\phi \in \mathscr{A}_{N} \\ y>0}}\left|\phi_{y} * f(x)\right|,
$$

the grand maximal function of $f$ of order $N$.

In this paper we prove weighted norm inequalities for the form

$$
\int\left|G_{N}(f)\right|^{p} V d x \leq C \int S_{\psi, \alpha}^{p}(f) \widetilde{M} V d x \quad(0<p<\infty) ;
$$

i.e., for each $0<p<\infty$ we exhibit a "maximal operator" (or class of operators) $\widetilde{M}$ such that (2) holds for all $f$ in some suitable test class (e.g., $\mathscr{C}_{0}^{\infty}\left(\mathbf{R}^{d}\right)$ or $\left.L^{p}\left(\mathbf{R}^{d}, d x\right)\right)$ and all nonnegative $V \in L_{\text {loc }}^{1}\left(\mathbf{R}^{d}\right)$, for appropriate $\psi$, and $N$ and $\alpha$ large enough, with a constant $C$ which does not depend on $V$ or $f$. The $M$ 's we obtain are smaller than any previously known, and in particular they do not (in general) have the Muckenhoupt $A_{\infty}$ property. (Recall that a weight $V$ is said to have $A_{\infty}$ if for all $\varepsilon>0$ there is a $\delta>0$ so that for all cubes $Q$ and subsets $E \subset Q$,

$$
\frac{|E|}{|Q|}<\delta \Rightarrow \int_{E} V<\varepsilon \int_{Q} V
$$

See [M].) We show that, for every $p$, there is a $k$ so that (2) holds for $\widetilde{M}=$ $M^{k}$, where $M$ is the Hardy-Littlewood maximal operator; moreover, when $0<p<2$, we can take $k=1$.

We apply our results in two directions. When $1<p \leq 2$, an additional argument lets us infer from (2) a new sufficient condition on weights $V$ and $W$ for the Sobolev inequality

$$
\int|f|^{p} V d x \leq \int|\nabla f|^{p} W d x
$$

to hold for all $f \in \mathscr{C}_{0}^{\infty}\left(\mathbf{R}^{d}\right)$. When $p=2$ we use this inequality to extend results of $[\mathrm{F}, \mathrm{CW} 1$, and $\mathrm{CWW}$ ], obtaining new eigenvalue estimates for Schrödinger operators of the form $L=-\operatorname{div}(A(x) \nabla \cdot)-V$, where $A(x)$ is a symmetric, positive semidefinite, matrix-valued function of $x \in \mathbf{R}^{d}$.

When $1<p<\infty$, (2) plus results from [CW2] let us obtain new weighted norm inequalities for Calderón-Zygmund operators. Let $\Omega \in \mathscr{C}^{\infty}\left(\mathbf{R}^{d} \backslash\{0\}\right)$ be homogeneous of degree 0 , and satisfy

$$
\int_{|x|=1} \Omega(x) d \sigma(x)=0 .
$$

If, for $f \in \bigcup_{1 \leq p<\infty} L^{p}\left(\mathbf{R}^{d}, d x\right)$, we set

$$
T f(x)=\lim _{\varepsilon \rightarrow 0} \int_{|y|>\varepsilon} f(x-y) \frac{\Omega(y)}{|y|^{d}} d y \quad \text { a.e. }
$$


then $T$ is called a Calderón-Zygmund operator (see [St]). We show that, if $1<p<2$, then

$$
\int|T f|^{p} V d x \leq C \int|f|^{p} M(M V) d x
$$

for all $f$ and all weights $V$. We obtain analogous, but more complicated, results for $2 \leq p<\infty$.

Our theorems are based on results in [W2, W3], and the main idea of this paper is the construction of a "machine" which allows us to translate the "dyadic" results of those papers into the continuous setting. We describe the machine in $\S 2$. Once the machine is in place, the Sobolev and singular integral inequalities follow relatively (though not quite) routinely. In particular, in order to obtain (2), it is enormously convenient to assume, at one point, an additional hypothesis on $\psi$; namely, that $\psi$ is not too smooth. Using the methods of [FS2], one can do without this assumption, but doing so does not seem to result in any stronger theorems in the applications. Therefore we have stuck with our assumption. However, in $\S 6$, we show how one may obtain the analogues of our square functions results without it.

We prove all the square function results we need in $\S 2$. In $\S 3$ we prove the results on Calderón-Zygmund operators. We prove the Sobolev inequalities in $\S 4$ and the eigenvalue estimates are proved in $\S 5$. In $\S 7$ we tie up a loose end which we leave hanging in $\S 3$, which is peripheral to the line of our argument there.

\section{SQUARE FUNCTION RESULTS}

We shall say that a cube $Q \subset \mathbf{R}^{d}$ is dyadic if it is of the form

$$
Q=\left(\frac{j_{1}}{2^{k}}, \frac{j_{1}+1}{2^{k}}\right) \times \cdots \times\left(\frac{j_{d}}{2^{k}}, \frac{j_{d}+1}{2^{k}}\right)
$$

for some integers $k$ and $j_{i}, i=1, \ldots, d$. Dyadic cubes have the well-known property that any two of them are either disjoint, or one of them is contained in the other. We shall denote the collection of all dyadic cubes by $\mathscr{D}$. If $Q$ is as above we say that $Q$ has sidelength $2^{-k}$, and we denote this by $l(Q)=2^{-k}$. For $f \in L_{\text {loc }}^{1}\left(\mathbf{R}^{d}\right)$ and $Q$ a cube, we set

$$
f_{Q} \equiv \frac{1}{|Q|} \int_{Q} f,
$$

the average value of $f$ over $Q$. For $k$ an integer we set

$$
f_{k} \equiv \sum_{\substack{Q \in \mathscr{D} \\ l(Q)=2^{-k}}} f_{Q} \chi_{Q} ;
$$

and we define

$$
f^{*}(x) \equiv \sup _{k}\left|f_{k}\right|,
$$


the dyadic maximal function of $f$. If $Q$ is a dyadic cube and $l(Q)=2^{-k}$ we define

$$
a_{Q}(f) \equiv\left(f_{k+1}-f_{k}\right) \chi_{Q} .
$$

The dyadic square function is defined by

$$
S(f) \equiv\left(\sum_{x \in Q \in \mathscr{D}} \frac{\left\|a_{Q}(f)\right\|_{2}^{2}}{|Q|}\right)^{1 / 2} .
$$

In this section we show how to reduce the study of $G_{N}$ and $S_{\psi, \alpha}$ to that of $f^{*}$ and $S$. In order to avoid getting lost in technicalities, we shall first prove our theorems under the assumption that $f \in \mathscr{C}_{0}^{\infty}\left(\mathbf{R}^{d}\right)$; at the end (of this section) we shall show how to remove this hypothesis.

The main device in our proof is the following lemma. By the triple of a cube, we mean the cube concentric with it but with sidelength three times as big. We shall always denote the triple of a given cube $Q$ by $\widetilde{Q}$.

Lemma 2.1. Let $\mathscr{F}$ be the collection of all triples of dyadic cubes. There exist disjoint families $\mathscr{G}_{1}, \ldots, \mathscr{G}_{3^{d}}$ such that $\mathscr{F}=\bigcup \mathscr{G}_{k}$, and, for every $k$, if $Q, Q^{\prime}$ are in $\mathscr{G}_{k}$, then either they are disjoint or one is contained in the other. Moreover, if $Q$ is a proper subset of $Q^{\prime}$, then $l(Q) \leq \frac{1}{2} l\left(Q^{\prime}\right)$.

Remark. This is a refinement of Lemma 3.2 from [CWW]. Also, it is implicit in work of Carleson and Garnett on interpolating sequences in $\mathbf{R}_{+}^{d+1}[G, p .416]$.

Proof. For $\vec{\varepsilon}=\left(\varepsilon_{1}, \ldots, \varepsilon_{d}\right) \in\{0,1,2\}^{d}$ and $k$ an integer, let $\mathscr{H}_{k}(\vec{\varepsilon})$ be those $Q$ in $\mathscr{F}$ which are of the form

$$
Q=\left(\frac{3 n_{1}+\varepsilon_{1}}{2^{k}}, \frac{3\left(n_{1}+1\right)+\varepsilon_{1}}{2^{k}}\right) \times \cdots \times\left(\frac{3 n_{d}+\varepsilon_{d}}{2^{k}}, \frac{3\left(n_{d}+1\right)+\varepsilon_{d}}{2^{k}}\right)
$$

for some integers $n_{1}, \ldots, n_{d}$. It is clear that $\mathscr{H}_{k}(\vec{\varepsilon}) \cap \mathscr{H}_{k}(\vec{\delta})=\varnothing$ if $\vec{\varepsilon} \neq \vec{\delta}$.

If we bisect a $Q$ in $\mathscr{H}_{k}(\vec{\varepsilon})$ into $2^{d}$ congruent subcubes, the resulting cubes belong to $\mathscr{H}_{k+1}(2 \vec{\varepsilon})$, where we are slightly abusing notation, and saying that $\varepsilon_{i}=\varepsilon_{i}^{*}$ if they are congruent modulo 3 . Also, every such $Q$ arises from the subdivision of a $Q^{\prime} \in \mathscr{H}_{k-1}(2 \vec{\varepsilon})$. Let us show this. It is sufficient to show that every interval $J=(3 n+\varepsilon, 3 n+3+\varepsilon)$ is either the right or left half of an interval $\left(6 n^{\prime}+2 \varepsilon^{\prime}, 6 n^{\prime}+6+2 \varepsilon^{\prime}\right)$, with $\varepsilon^{\prime}=2 \varepsilon$. We consider cases:

(i) $\varepsilon=0$. If $n=2 k$ then take $n^{\prime}=k, \varepsilon^{\prime}=0$, and $J$ is a left half. If $n=2 k+1$ then take $n^{\prime}=k, \varepsilon^{\prime}=0$, and $J$ is a right half.

(ii) $\varepsilon=1$. If $n=2 k$, take $n^{\prime}=k, \varepsilon^{\prime}=2$, and $J$ is a right half. If $n=2 k+1$, take $n^{\prime}=k, \varepsilon^{\prime}=2$, and $J$ is a left half.

(iii) $\varepsilon=2$. If $n=2 k$, take $n^{\prime}=k, \varepsilon^{\prime}=1$ (which equals $2 \cdot 2$ modulo 3 ), and $J$ is a left half. If $n=2 k+1$, take $n^{\prime}=k, \varepsilon^{\prime}=1$, and $J$ is a right half.

Therefore if we set

$$
\mathscr{G}(\vec{\varepsilon})=\bigcup_{k} \mathscr{H}_{k}\left(2^{|k|} \vec{\varepsilon}\right),
$$

then these are the desired collections. Q.E.D. 
For the time being, let us assume that $f \in \mathscr{C}_{0}^{\infty}\left(\mathbf{R}^{d}\right)$, and let $\psi$ be as in the introduction (in particular, $\psi$ satisfies $(*)$ ); at the end of this section we shall show how to extend our results to general $f \in \bigcup_{0<p<\infty} H^{p}\left(\mathbf{R}^{d}, d x\right)$, where $H^{p}$ denotes the Fefferman-Stein real-variable Hardy space, as defined in [FS2]. Let $\mathbf{R}_{+}^{d+1}=\left\{(t, y): t \in \mathbf{R}^{d}, y>0\right\}$. We use A. P. Calderón's trick to write

$$
f(x)=\int_{\mathbf{R}_{+}^{d+1}} f * \psi_{y}(t) \psi_{y}(x-t) \frac{d t d y}{y} .
$$

Since $f \in \mathscr{C}_{0}^{\infty}\left(\mathbf{R}^{d}\right)$, the integral converges absolutely, uniformly for all $x$, and so we can cut it up however we please.

For $Q$ a dyadic cube define

$$
T(Q) \equiv\left\{(t, y) \in \mathbf{R}_{+}^{d+1}: t \in Q, \frac{1}{2} l(Q) \leq y<l(Q)\right\}
$$

and set

$$
\tilde{a}_{Q}(f)=\int_{T(Q)} f * \psi_{y}(t) \psi_{y}(x-t) \frac{d t d y}{y} .
$$

Clearly each $\tilde{a}_{Q}(f)$ is supported in $\widetilde{Q}$, is smooth, and has integral 0 . Also,

$$
f=\sum_{Q} \tilde{a}_{Q}(f) .
$$

For $1 \leq k \leq 3^{d}$, let $\mathscr{G}_{k}$ be one of the collections obtained in Lemma 2.1. We set

$$
f_{(k)} \equiv \sum_{\widetilde{Q} \in \mathscr{G}_{k}} \tilde{a}_{Q}(f)
$$

and we have

$$
f=\sum_{k=1}^{3^{d}} f_{(k)} .
$$

The $f_{(k)}$ 's are sums of smooth functions which behave very much like the dyadia martingale differences $a_{Q}(f)$. We shall make this precise with the following definitions. Let us say that a collection of cubes $\mathscr{G}$ is good if for all $Q, Q^{\prime} \in \mathscr{G}$, either $Q \cap Q^{\prime}=\varnothing$ or else one is contained in the other, and $Q \subset Q^{\prime}, Q \neq Q^{\prime}$ implies $l(Q) \leq \frac{1}{2} l\left(Q^{\prime}\right)$. Given a cube $Q$, we will say that a function $a(x)$ is adapted to $Q$ if $\operatorname{supp} a \subset Q,\|a\|_{\infty} \leq|Q|^{-1 / 2},\|\nabla a\|_{\infty} \leq l(Q)^{-1}|Q|^{-1 / 2}$, and $\int a=0$. (This is different from the usual definition of an adapted function, as in [GJ], where one requires $\operatorname{supp} a \subset \widetilde{Q}$.) Finally, we will say that a function $f$ is of special form if there exist a good collection $\mathscr{G}$, functions $a_{Q}(x)$ adapted to $Q \in \mathscr{G}$, and constants $\lambda_{Q}$ such that

$$
f=\sum_{Q \in \mathscr{G}} \lambda_{Q} a_{Q} .
$$

We will say that such an $f$ is of special form relative to $\mathscr{G}$. 
Note that each $f_{(k)}$ is of special form relative to $\mathscr{G}_{k}$, where, for $Q \in \mathscr{D}$, $\widetilde{Q} \in \mathscr{G}_{k}$, we can take

$$
\mid \lambda \widetilde{Q}^{\mid} \leq C(\psi, d)\left(\int_{T(Q)}\left|f * \psi_{y}(t)\right|^{2} \frac{d t d y}{y}\right)^{1 / 2} .
$$

Whenever we deal with a sum like (3), it will be obvious that it converges absolutely, uniformly for all $x$. Also, the family $\mathscr{G}$ will always be a subset of one of our collections $\mathscr{G}_{k}$ from the lemma. Since the only properties we will use about the $Q \in \mathscr{G}$ are those entailed in the definition of goodness, it will never be any loss of generality to assume that $\mathscr{G}$ is a subset of $\mathscr{D}$.

Now, let $\mathscr{G} \subset \mathscr{D}$ and let $f$ be as in (3). We define

$$
S_{\Lambda}(f) \equiv\left(\sum_{x \in Q \in \mathscr{G}} \frac{\left|\lambda_{Q}\right|^{2}}{|Q|}\right)^{1 / 2}
$$

The function $S_{\Lambda}(f)$ "looks like" the dyadic square function $S(f)$. The following two lemmas explain how these functions are related.

Lemma 2.2. Let $f$ and $\mathscr{G}$ be as above. There is a $C(d)$ such that

$$
S(f) \leq C(d) S_{\Lambda}(f)
$$

for all $x \in \mathbf{R}^{d}$.

Proof. To avoid confusion, we shall denote the adapted functions in (3) by $b_{Q}$.

Let $Q \in \mathscr{D}$. We need to estimate $\left\|a_{Q}(f)\right\|_{2}$. Because the $b_{Q}$ 's have integral 0 , we have

$$
a_{Q}(f)=\sum_{Q \subset Q^{\prime} \in \mathscr{G}} \lambda_{Q^{\prime}} a_{Q}\left(b_{Q^{\prime}}\right)
$$

Now, the smoothness of the $b_{Q}$ 's implies

$$
\left|a_{Q^{(}}\left(b_{Q^{\prime}}\right)\right| \leq C(d)\left|Q^{\prime}\right|^{-1 / 2} l\left(Q^{\prime}\right)^{-1} l(Q) .
$$

Therefore,

$$
\left\|a_{Q}(f)\right\|_{2}^{2} \leq C(d) \sum_{Q \subset Q^{\prime} \in \mathcal{G}}\left|\lambda_{Q^{\prime}}\right|^{2} \frac{|Q|}{\left|Q^{\prime}\right|}\left(\frac{l(Q)}{l\left(Q^{\prime}\right)}\right) .
$$

This implies that

$$
\begin{aligned}
& \sum_{x \in Q \in \mathscr{Q}} \frac{\left\|a_{Q}(f)\right\|_{2}^{2}}{|Q|} \leq C(d) \sum_{x \in Q \in \mathscr{D}} \sum_{Q \subset Q^{\prime} \in \mathscr{G}} \frac{\left|\lambda_{Q^{\prime}}\right|^{2}}{\left|Q^{\prime}\right|} \frac{l(Q)}{l\left(Q^{\prime}\right)} \\
& =C(d) \sum_{x \in Q^{\prime} \in \mathscr{G}} \frac{\left|\lambda_{Q^{\prime}}\right|^{2}}{\left|Q^{\prime}\right|} \sum_{\substack{x \in Q \subset Q^{\prime} \\
Q^{\prime} \in \mathscr{S}}} \frac{l(Q)}{l\left(Q^{\prime}\right)} \\
& \leq C(d) \sum_{x \in Q^{\prime} \in \mathscr{G}} \frac{\left|\lambda_{Q^{\prime}}\right|^{2}}{\left|Q^{\prime}\right|}
\end{aligned}
$$

which is (4). Q.E.D. 
Remark. We shall need the following facts in $\S 5$, when we deal with Schrödinger operators. For $Q$ a dyadic cube, let $\tilde{f}_{Q}$ be the $L^{2}$ projection of $f$ onto those functions which are linear + constant on $Q$. Define, for $k$ an integer,

$$
\tilde{f}_{k} \equiv \sum_{l(Q)=2-k} \tilde{f}_{Q} \chi_{Q}
$$

and set $\tilde{a}_{Q}(f) \equiv\left(\tilde{f}_{k+1}-\tilde{f}_{k}\right) \chi_{Q}$, for $Q \in \mathscr{D}, l(Q)=2^{-k}$. We notice that $\tilde{a}_{Q}(f)$ is linear + constant on each of the immediate dyadic subcubes of $Q$, and is orthogonal to any function which is linear + constant on all of $Q$. Define

$$
\tilde{S}^{2}(f) \equiv \sum_{x \in Q} \frac{\left\|\tilde{a}_{Q}(f)\right\|_{2}^{2}}{|Q|} .
$$

This is the square function which Fefferman uses in [F].

We claim that $S(f) \leq C(d) \widetilde{S}(f)$. We need to estimate $a_{Q}(f)$. Since the $\tilde{a}_{Q}(f)$ 's have integral 0 , that is the same as

$$
a_{Q}\left(\sum_{Q \subset Q^{\prime}} \tilde{a}_{Q^{\prime}}(f)\right),
$$

so what we really need to estimate is $a_{Q}\left(\tilde{a}_{Q^{\prime}}(f)\right)$ for $Q \subset Q^{\prime}$.

It is obvious that $\left|a_{Q}\left(\tilde{a}_{Q}(f)\right)\right| \leq C\left\|\tilde{a}_{Q}(f)\right\|_{\infty}$. The other $\tilde{a}_{Q^{\prime}}(f)$ 's are linear across $Q$, so it is easy to see that

$$
\left|a_{Q^{\prime}}\left(\tilde{a}_{Q^{\prime}}(f)\right)\right| \leq C\left\|\tilde{a}_{Q^{\prime}}(f)\right\|_{\infty} \frac{l(Q)}{l\left(Q^{\prime}\right)} .
$$

Therefore,

$$
\begin{aligned}
\frac{\left\|a_{Q}(f)\right\|_{2}^{2}}{|Q|} & \leq C \sum_{Q \subset Q^{\prime}}\left\|\tilde{a}_{Q^{\prime}}(f)\right\|_{\infty}^{2} \frac{l(Q)}{l\left(Q^{\prime}\right)} \\
& \leq C \sum_{Q \subset Q^{\prime}} \frac{\left\|\tilde{a}_{Q^{\prime}}(f)\right\|_{2}^{2}}{\left|Q^{\prime}\right|} \frac{l(Q)}{l\left(Q^{\prime}\right)},
\end{aligned}
$$

and now summing over $Q \ni x$ yields the result.

This means that all of our inequalities for $S$ work just as well for $\widetilde{S}$.

The other fact we will need is this. Suppose that the adapted functions $b_{Q}$ in Lemma 2.2 are constructed to satisfy $\int b_{Q} P(x) d x=0$ for all polynomials $P$ with degree $\leq 1$, and $\left\|D^{\alpha} b_{Q}\right\|_{\infty} \leq|Q|^{-1 / 2} l(Q)^{-2}$ for all $|\alpha|=2$. Then

$$
\sum_{x \in Q} \frac{\left\|\tilde{a}_{Q}(f)\right\|_{2}^{2}}{|Q|} l(Q)^{-2} \leq C(d) \sum_{x \in Q} \frac{\left|\lambda_{Q}\right|^{2}}{|Q|} l(Q)^{-2} .
$$

The proof is almost exactly like that of Lemma 2.2. It uses the fact that $\tilde{a}_{Q}\left(b_{Q^{\prime}}\right) \equiv 0$ if $Q \not \subset Q^{\prime}$ (this is because of $b_{Q^{\prime}}$ 's extra cancellation) and 
$\left|\tilde{a}_{Q}\left(b_{Q^{\prime}}\right)\right| \leq C(d)\left|Q^{\prime}\right|^{-1 / 2}\left(l(Q) / l\left(Q^{\prime}\right)\right)^{2}$ when $Q \subset Q^{\prime}$ (because of $b_{Q^{\prime}}$ 's extra smoothness and the definition of $\tilde{a}_{Q}$ ).

Our next lemma is the continuous version of a somewhat strengthened form of the (one and only) lemma from [W3]. In [W1] we defined the functional $Y(Q, V)$ for cubes $Q$ and nonnegative $V \in L_{\mathrm{loc}}^{1}\left(\mathbf{R}^{d}\right)$ as follows:

$$
Y(Q, V) \equiv \begin{cases}\frac{\int_{Q} M\left(\chi_{Q} V\right)}{\int_{Q} V}, & \int_{Q} V>0, \\ 1, & \int_{Q} V=0 .\end{cases}
$$

This functional measures the "peakiness" (un- $A_{\infty}$ behavior) of $V$ on $Q$ : $Y(Q, V)$ is large if, relative to $Q, V$ has most of its mass concentrated on a small set. In our next lemma we shall make use of a slightly less singular version of $Y(Q, V)$. For $0<\eta \leq 1$, and $Q$ and $V$ as above, define

$$
Y_{\eta}(Q, V) \equiv \begin{cases}\frac{\int_{Q} V(x) \log ^{\eta}\left(e+V(x) / V_{Q}\right) d x}{\int_{Q} V d x}, & \int_{Q} V>0, \\ 1, & \int_{Q} V=0 .\end{cases}
$$

Note that $Y_{1}(Q, V) \sim Y(Q, V)$, i.e., their ratio is bounded above and below by constants that depend only on $d$ [St, p. 23].

Lemma 2.3. Let $0<p<\infty, 0<\eta \leq 1$, and let $A$ be a positive number. Let $\mathscr{G} \subset \mathscr{D}$. Let $f$ be as in (3), and such that $f^{*} \in L^{p}\left(\mathbf{R}^{d}, V d x\right)$. Suppose that $V$ is a weight for which $Y_{\eta}(Q, V) \leq A$ for all $Q \in \mathscr{G}$. Then there is a $C(p, d, \eta)<\infty$ such that

$$
\int\left|f^{*}\right|^{p} V d x \leq C(p, d, \eta) A^{p / 2 \eta} \int S_{\Lambda}^{p}(f) V d x .
$$

Remark. Essentially the same proof as the one given below shows that the dyadic version of this lemma also holds.

Proof. Let $V\{\cdots\}$ denote the $V d x$ measure of the set $\{\cdots\}$. It is enough to show that, for all $\lambda>0$,

$$
V\left\{f^{*}>2 \lambda, S_{\Lambda}(f) \leq \gamma \lambda\right\} \leq \varepsilon(p) V\left\{f^{*}>\lambda\right\}
$$

for appropriate $\varepsilon(p)$, and with $\gamma>C(p, d, \eta) A^{-1 / 2 \eta}$.

Let $\left\{Q_{\lambda}^{i}\right\}$ be the maximal dyadic cubes such that $\left|f_{Q_{i}^{\prime}}\right|>\lambda$. It is enough to show that

$$
V\left\{x \in Q_{\lambda}^{i}: f^{*}>2 \lambda, S_{\Lambda}(f) \leq \gamma \lambda\right\} \leq \varepsilon(p) V\left(Q_{\lambda}^{i}\right)
$$

for all $Q_{\lambda}^{i}$ such that

$$
\sum_{\substack{Q ! \subset Q \in \mathscr{G} \\ Q_{i}^{\prime} \neq Q}} \frac{\left|\lambda_{Q}\right|^{2}}{|Q|} \leq \gamma^{2} \lambda^{2}
$$


An immediate consequence of (5) - and the size and smoothness condition on the adapted functions $a_{Q}$-is that we may assume $\left|f_{Q_{i}}\right| \leq(1.1) \lambda$.

Let $\left\{Q_{k}\right\}$ be maximal (and not necessarily proper) subcubes of $Q_{\lambda}^{i}$ which belong to $\mathscr{G}$. Suppose $Q \in \mathscr{D}$ satisfies $Q \subset Q_{\lambda}^{i}$, but $Q \not \subset Q_{k}$ for any $k$. A moment's thought shows that we have $\left|f_{Q}-f_{Q_{\lambda}^{i}}\right| \leq C(d) \gamma \lambda$. Therefore, if we take $\gamma$ small enough, the set we are trying to bound will be contained in

$$
\bigcup_{k}\left\{x \in Q_{k}:\left(f-f_{Q_{k}}\right)^{*}>(.8) \lambda, S_{\Lambda}(f) \leq \gamma \lambda\right\} \equiv \bigcup_{k} E_{k}
$$

But Lemma 2.2 says that $S(f) \leq C(d) S_{\Lambda}(f)$. Therefore, Theorem 3.1 from [CWW] says that, for each $k$,

$$
\frac{\left|E_{k}\right|}{\left|Q_{k}\right|} \leq B \exp \left(-C \gamma^{-2}\right),
$$

where $B$ and $C$ are positive constants that depend on $d$. Since $Y_{\eta}\left(Q_{k}, V\right) \leq$ $A$, we will have $V\left(E_{k}\right) \leq \varepsilon(p) V\left(Q_{k}\right)$ if we take $\gamma \sim A^{-1 / 2 \eta}$. This finishes the proof. Q.E.D.

The next lemma is a slightly strengthened form of Lemma 2 from [W2]. Before stating it we need another definition. Let $\rho:[1, \infty) \rightarrow[1, \infty)$ be increasing and satisfy $\rho(2 x) \leq A \rho(x)$ for all $x$. We define

$$
M_{\rho} V(x) \equiv \sup _{x \in Q} \rho(Y(Q, V)) V_{Q} .
$$

Our lemma is

Lemma 2.4. Let $\rho$ be as above. There is a $C(A, d)$ so that for all nonnegative weights $V$ and all cubes $Q$,

$$
\rho(Y(Q, V)) \int_{Q} M\left(\chi_{Q} V\right) d x \leq C(A, d) \int_{Q} M_{\rho}\left(\chi_{Q} V\right) d x .
$$

Proof. It is enough to prove the lemma for $Q$ a dyadic cube and $M$ the dyadic Hardy-Littlewood maximal function.

Let $Q_{i} \subset Q$ be the maximal dyadic subcubes such that

$$
Y\left(Q_{i}, V\right) \leq \frac{1}{2} Y(Q, V) .
$$

Define

Clearly,

$$
\widetilde{V}(x) \equiv \begin{cases}V_{Q_{i}}, & x \in Q_{i}, \\ V(x), & x \notin \cup Q_{i} .\end{cases}
$$

$$
\begin{aligned}
\int_{Q} M\left(\chi_{Q} V\right) d x & \leq \int_{Q} M\left(\chi_{Q} \widetilde{V}\right) d x+\sum_{i} \int_{Q_{i}} M\left(\chi_{Q_{i}} V\right) d x \\
& =\int_{Q} M\left(\chi_{Q} \widetilde{V}\right) d x+\sum_{i} Y\left(Q_{i}, V\right) V\left(Q_{i}\right) \\
& \leq \int_{Q} M\left(\chi_{Q} \widetilde{V}\right) d x+\frac{1}{2} Y(Q, V) V(Q) .
\end{aligned}
$$


But the left-hand side of $(6)$ is just $Y(Q, V) V(Q)$; therefore,

$$
\int_{Q} M\left(\chi_{Q} V\right) d x \leq 2 \int_{Q} M\left(\chi_{Q} \widetilde{V}\right) d x
$$

It is obvious that

$$
\int_{Q} M\left(\chi_{Q} \widetilde{V}\right) d x \leq 2^{d} \int_{\substack{Q \in Q^{*} \subset Q \\ Q^{*} \not \subset \bigcup Q_{i}}} \sup _{Q^{*}} d x
$$

All of the cubes in the "sup" satisfy $Y\left(Q^{*}, V\right) \geq \frac{1}{2} Y(Q, V)$. Combining this with (7) and $\rho$ 's doubling condition yields the lemma. Q.E.D.

We are now ready to prove our main square function results. Let us fix a function $\psi$ as in the introduction. We shall prove two theorems:

Theorem 2.5. Let $0<p<2$ and $p / 2<\eta \leq 1$. Let $V$ and $W$ be nonnegative weights such that

$$
\int_{Q} V(x) \log ^{\eta}\left(e+\frac{V(x)}{V_{Q}}\right) d x \leq \int_{Q} W(x) d x
$$

for all cubes $Q$. There is a $C(p, d, \eta)<\infty$ so that for all $f \in \mathscr{C}_{0}^{\infty}\left(\mathbf{R}^{d}\right)$ and all $\alpha \geq 3 \sqrt{d}$,

$$
\int|f|^{p} V d x \leq C(p, d, \eta) \int S_{\psi, \alpha}^{p}(f) W d x .
$$

This theorem has an immediate consequence.

Corollary 2.6. Let $0<p<2$ and $\alpha>3 \sqrt{d}$. There is a $C(p, d)$ such that

$$
\int|f|^{p} V d x \leq C(p, d) \int S_{\psi, \alpha}^{p}(f) M V d x
$$

for all $f \in \mathscr{C}_{0}^{\infty}\left(\mathbf{R}^{d}\right)$ and weights $V$, where $M$ is the Hardy-Littlewood maximal operator.

Proof of Corollary 2.6. By an easy limiting argument, we can assume that $V$ is bounded. Then apply the previous theorem with $\eta=1$ and $W=M V$. Q.E.D.

Theorem 2.7. Let $2 \leq p<\infty$. Let $\mathrm{\aleph}:[0, \infty) \rightarrow[1, \infty)$ be increasing and satisfy $\aleph(2 x) \leq A \aleph(x)$ for all $x$. Assume that

$$
\sum_{k=0}^{\infty} \aleph(k)^{-1 /(p-1)} \leq 1
$$

Set $\rho(x) \equiv \aleph(\log x) x^{p / 2-1}$. Let $V$ and $W$ be weights such that

$$
\int_{Q} M_{\rho}\left(\chi_{Q} V\right) d x \leq \int_{Q} W d x
$$

for all cubes $Q$. There is a $C(p, d, A)<\infty$ such that

$$
\int|f|^{p} V d x \leq C(p, d, A) \int S_{\psi, \alpha}^{p}(f) W d x
$$

for all $f \in \mathscr{C}_{0}^{\infty}\left(\mathbf{R}^{d}\right)$ and $\alpha \geq 3 \sqrt{d}$. 
Proof of Theorem 2.5. Let $\mathscr{G}_{k}$ be the collections obtained in Lemma 2.1, and write

$$
f=\sum_{k} f_{(k)}
$$

where each $f_{(k)}$ is of special form relative to $\mathscr{G}_{k}$.

Let us temporarily fix $k$, setting $f \equiv f_{(k)}$. For $j$ a nonnegative integer define

$$
\mathscr{F}_{j} \equiv\left\{Q \in \mathscr{G}_{k}: 2^{j} \leq Y_{\eta}(Q, V)<2^{j+1}\right\}
$$

Set

$$
f_{j} \equiv \sum_{Q \in \mathscr{F}_{j}} \lambda_{Q} a_{Q}
$$

where the $a_{Q}$ are the adapted functions. Define

$$
c_{Q^{\prime}}\left(f_{j}\right) \equiv\left(\sum_{Q^{\prime} \subseteq Q \in \mathscr{F}_{j}} \frac{\left|\lambda_{Q}\right|^{2}}{|Q|}\right)^{p / 2}-\left(\sum_{\substack{Q^{\prime} \subset Q \in \mathscr{F}_{j} \\ Q^{\prime} \neq Q}} \frac{\left|\lambda_{Q}\right|^{2}}{|Q|}\right)^{p / 2}
$$

for any $Q^{\prime} \in \mathscr{G}_{k}$. Observe that $S_{\Lambda}^{p}\left(f_{j}\right)=\sum_{x \in Q} c_{Q}\left(f_{j}\right)$, and that $c_{Q}\left(f_{j}\right)=0$ if $Q \notin \mathscr{F}_{j}$. Let $f^{*}$ denote the "dyadic" maximal function, relative to $\mathscr{G}_{k}$, of $f$. And write

$$
\begin{aligned}
\int\left|f^{*}\right|^{p} V d x & \leq C \sum_{j}(1+j)^{2} \int\left|f_{j}^{*}\right|^{p} V d x \\
& \leq C(p, d, \eta) \sum_{j}(1+j)^{2} 2^{j p / 2 \eta} \int S_{\Lambda}^{p}\left(f_{j}\right) V d x \\
& =C(p, d, \eta) \sum_{j}(1+j)^{2} 2^{j p / 2 \eta} \int \sum_{x \in Q \in \mathscr{F}_{j}} c_{Q}\left(f_{j}\right) V d x \\
& =C(p, d, \eta) \sum_{j}(1+j)^{2} 2^{j p / 2 \eta} \sum_{Q \in \mathscr{F}_{j}} c_{Q}\left(f_{j}\right) V(Q) \\
& \leq C(p, d, \eta) \sum_{j}(1+j)^{2} 2^{j(p / 2 \eta-1)} \sum_{Q \in \mathscr{F}_{j}} c_{Q}\left(f_{j}\right) W(Q) \\
& =C(p, d, \eta) \sum_{j}(1+j)^{2} 2^{j(p / 2 \eta-1)} \int S_{\Lambda}^{p}\left(f_{j}\right) W d x \\
& \leq C(p, d, \eta) \int S_{\Lambda}^{p}(f) W d x,
\end{aligned}
$$

where (8) is from Lemma $2.3,(9)$ is from the definition of $Y_{\eta}(Q, V)$ and the hypothesis on $V$ and $W$, and (10) is from the fact that $p / 2 \eta-1<0$.

We have thus proved the theorem for each $S_{\Lambda}\left(f_{(k)}\right)$. But is is obvious that $\sum S_{\Lambda}\left(f_{(k)}\right) \leq C(d) S_{\psi, \alpha}(f)$ if $\alpha \geq 3 \sqrt{d}$. This finishes the proof. Q.E.D. 
Proof of Theorem 2.7. Let us fix $k$ as before. After setting $\eta=1$, let us define $\mathscr{F}_{j}, f_{j}$, and $c_{Q}\left(f_{j}\right)$ as we did above. We write

$$
\begin{aligned}
\int\left|f^{*}\right|^{p} V d x & \leq \sum_{j} \aleph(j) \int\left|f_{j}^{*}\right|^{p} V d x \\
& \leq C(p, d) \sum_{j} \aleph(j) 2^{j p / 2} \int S_{\Lambda}^{p}\left(f_{j}\right) V d x \\
& =C(p, d) \sum_{j} \aleph(j) 2^{j p / 2} \sum_{Q \in \mathscr{F}_{j}} c_{Q}\left(f_{j}\right) V(Q) \\
& \leq C(p, d, A) \sum_{j} \sum_{Q \in \mathscr{F}_{j}} c_{Q}\left(f_{j}\right) \int_{Q} M_{\rho}\left(\chi_{Q} V\right) d x \\
& \leq C(p, d, A) \sum_{Q \in \mathscr{F}_{k}} c_{Q}(f) W(Q) \\
& \leq C(p, d, A) \int S_{\Lambda}^{p}(f) W d x
\end{aligned}
$$

where the next-to-last line follows from the fact that $p \geq 2$, and line (11) is because of Lemma 2.4. The theorem is proved. Q.E.D.

The maximal function $M_{\rho}$, at first sight, looks quite bizarre. However, it is not so bad as it appears. In fact, it is less than or equal to $C(p, d, A) M^{k(\rho)} V$, where $M^{k}$ denotes the $k$-fold application of the Hardy-Littlewood maximal operator, $A$ is $\aleph$ 's doubling constant, and we define $k(p) \equiv[p / 2+1]$. We only need to prove this fact for $p>2$. Let us normalize $\aleph$ so that $\aleph(0)=1$. Observe that $\aleph(x) \leq C(A)(1+x)^{\gamma}$, for some positive $\gamma=\gamma(A)$, and therefore, for appropriate $0<\delta<1$ (large enough to ensure $p / 2-\delta<[p / 2]$ ),

$$
\begin{aligned}
M_{\rho} V(x) & \leq C(p, d, A) \sup _{x \in Q} Y(Q, V)^{p / 2-\delta} V_{Q} \\
& \leq C(p, d, A) \sup _{x \in Q} \frac{1}{|Q|} \int_{Q} M^{[p / 2]}\left(\chi_{Q} V\right) d t \\
& \leq C(p, d, A) M^{[p / 2+1]} V(x) \\
& =C(p, d, A) M^{k(p)} V(x),
\end{aligned}
$$

where we have used Hölder's inequality and the fact that

$$
\int_{Q} M^{k}\left(\chi_{Q} V\right) d x \sim \int_{Q} V(x) \log ^{k}\left(e+\frac{V(x)}{V_{Q}}\right) d x
$$

(see $[\mathrm{St}])$.

In Theorems 2.5 and 2.7, and Lemma 2.6, the left-hand sides of the asserted inequalities are all integrals in $|f|^{p}$. But in the introduction we claimed that we would get integrals in $\left|G_{N}(f)\right|^{p}$. We shall now show how to do this. It is here that we place an additional condition on $\psi$, which we shall assume holds for the rest of this section. 
We shall say that $\psi$ is rough enough if there are positive constants $C$ and $\beta$ so that

$$
\int_{s}^{\infty}|\hat{\psi}(t, 0, \ldots, 0)|^{2} \frac{d t}{t} \geq C(1+|s|)^{-\beta}
$$

for all $s>0$. We shall denote the left-hand side of (12) by $\boldsymbol{\Theta}(s)$.

Define

$$
H(x) \equiv \int_{1}^{\infty} \psi_{y} * \psi_{y}(x) \frac{d y}{y} .
$$

A computation shows that, as a distribution, $\widehat{H}(\xi)=\Theta(|\xi|)$. Therefore if we take $\psi$ sufficiently smooth (but still satisfying (12)) then $H$ will be a continuous function. We shall henceforth assume that $\psi$ is sufficiently smooth.

Suprisingly, the function $H$ has compact support (the first to observe this remarkable fact appears to be Uchiyama: see [U, p. 238]). The proof is, fortunately, quite simple. From $(*)$ we have that, as a distribution,

$$
H(x)=\delta_{0}-\int_{0}^{1} \psi_{y} * \psi_{y}(x) \frac{d y}{y},
$$

where $\delta_{0}$ is the Dirac mass at 0 . But the $x$-support of the integral is obviously contained in $\{|x| \leq 2\}$. Therefore supp $H \subset\{|x| \leq 2\}$.

The lemma we must now prove is

Lemma 2.8. Let $\psi$ be as above, and satisfy (12). There is an $N^{\prime}(\beta, d)$ such that, for all $N>N^{\prime}$,

$$
G_{N}(f)(x) \leq C(N, \beta, d) \sup _{|x-t|<3 y}\left|H_{y} * f(t)\right|
$$

for all $f \in L_{\text {loc }}^{1}\left(\mathbf{R}^{d}\right)$ and all $x \in \mathbf{R}^{d}$.

Proof. Let us take $f \in L_{\text {loc }}^{1}\left(\mathbf{R}^{d}\right)$. It is enough to show that

$$
|f * \phi(0)| \leq C(N, \beta, d) \sup _{|t|<3}|H * f(t)|
$$

for all $\phi \in \mathscr{A}_{N}$. So take $\phi \in \mathscr{A}_{N}$. If $N>\beta+d$ then there is a $g \in L^{2}\left(\mathbf{R}^{d}\right)$ (with $\|g\|_{2} \leq C(N, \beta, d)$ ) such that $\phi=g * H$, i.e.,

$$
\phi(x)=\int g(s) H(x-s) d s .
$$

Since $\operatorname{supp} \phi \subset\{|x| \leq 1\}$ and $\operatorname{supp} H \subset\{|x| \leq 2\}$, the integral in (13) is unchanged if we replace $g$ by $\tilde{g} \equiv g \cdot \chi_{\{|x| \leq 3\}}$. Therefore,

$$
\begin{aligned}
|f * \phi(0)| & =|f * H * \tilde{g}(0)| \\
& =\left|\int_{|s| \leq 3} f * H(-s) \tilde{g}(s) d s\right| \\
& \leq \sup _{|s| \leq 3}|f * H(s)|\|\tilde{g}\|_{1} \\
& \leq C(N, \beta, d) \sup _{|s| \leq 3}|f * H(s)| . \quad \text { Q.E.D. }
\end{aligned}
$$


Let us define

$$
H^{*}(f)(x) \equiv \sup _{|x-t|<3 y}\left|H_{y} * f(t)\right| .
$$

Given $f \in \mathscr{C}_{0}^{\infty}\left(\mathbf{R}^{d}\right)$, let $f_{(k)}$ be those functions obtained via Limma 2.1, and let $f_{(k)}^{*}$ be their corresponding "dyadic" maximal functions. Then, because of the preceding lemma, our grand maximal function results will follow immediately once we show that

$$
H^{*}(f) \leq C\left(\sum_{k=1}^{3^{d}} f_{k}^{*}+S_{\psi, \alpha}(f)\right)
$$

for $\alpha \geq 3 \sqrt{d}$.

We begin by observing that

$$
H_{y} * f(x)=\int_{\eta>y} f * \psi_{\eta}(t) \psi_{\eta}(x-t) \frac{d t d \eta}{\eta} .
$$

It is an easy consequence of (14) that, for any $(x, y) \in \mathbf{R}_{+}^{d+1}$,

$$
\begin{aligned}
& \sup _{|x-t|<3 y}\left|H_{y} * f(x)-H_{y} * f(t)\right| \leq C S_{\psi, \alpha}(f)(x), \\
& \sup _{y^{\prime} \leq y<2 y^{\prime}}\left|H_{y} * f(x)-H_{y^{\prime}} * f(x)\right| \leq C S_{\psi, \alpha}(f)(x)
\end{aligned}
$$

for $\alpha$ bigger than, say, 20. Therefore we only need to estimate $H^{+}(f)(x) \equiv$ $\sup _{-\infty<j<\infty}\left|H_{2^{j}} * f(x)\right|$. So we write

$$
\begin{aligned}
H_{2^{j}} * f(x) & =\int_{y>2^{j}} f * \psi_{y}(t) \psi_{y}(x-t) \frac{d t d y}{y} \\
& =\sum_{k=1}^{3^{d}} \sum_{\substack{Q \in \mathscr{G}_{k} \\
l(Q)>3^{j}}} \lambda_{Q^{j}} a_{Q}(x)
\end{aligned}
$$

where the $\lambda_{Q}$ and $a_{Q}$ are the appropriate constants and adapted functions which belong to the $f_{(k)}$. Fix $k$, and let $Q^{\prime}$ be the minimal cube in $\mathscr{G}_{k}$ such that $x \in Q^{\prime}$ and $l\left(Q^{\prime}\right)>3 \cdot 2^{j}$. The same sort of argument as the one in Lemma 2.2 shows that

$$
\left|\sum_{\substack{Q \in \mathscr{G}_{k} \\ Q^{\prime} \subseteq Q}} \lambda_{Q^{\prime}} a_{Q}(x)-\left(f_{(k)}\right)_{Q^{\prime}}\right| \leq C(d) S_{\Lambda}\left(f_{(k)}\right) .
$$

Therefore, the sum in (15) is dominated by

$$
C(d) \sum_{k=1}^{3^{d}}\left(f_{(k)}^{*}\right)+S_{\Lambda}\left(f_{(k)}\right) .
$$

This proves our inequality. The "grand maximal" versions of our results follow immediately. 
It is now easy to extend our results to arbitrary $f \in H^{p}$. First choose $\psi$ sufficiently smooth (depending on $p$ ), but still rough enough, so that $f * \psi_{y}(t)$ is well-behaved. Then, for $M$ a positive integer, define

$$
F^{(M)}(x) \equiv \int_{2^{-M} \leq y<2^{M}} f * \psi_{y}(t) \psi_{y}(x-t) \frac{d t d y}{y} .
$$

We can now decompose each $F^{(M)}$ into $3^{d}$ functions $f_{(k)}^{(M)}$, each of special form relative to $\mathscr{G}_{k}$. The adapted funtions we get will live on cubes with sidelengths between $3 \cdot 2^{-M}$ and $3 \cdot 2^{M}$, so there will be no problem about summing them up; and the cubes' disjointness properties imply that, if $Q \in \mathscr{G}_{k}$ and $l(Q)>3 \cdot 2^{M}$, then $\int_{Q} f_{(k)}^{(M)}=0$. This means that, for each $k$,

$$
\int\left|\left(f_{(k)}^{(M)}\right)^{*}\right|^{p} V d x=\sum_{\substack{Q \in \mathscr{F}_{k} \\ l(Q)=3^{M} 2^{M}}} \int_{Q}\left|\left(f_{(k)}^{(M)} \chi_{Q}\right)^{*}\right|^{p} V d x,
$$

and each integral in the sum is finite. Now we only need to apply our previous arguments, let $M \rightarrow \infty$, and apply Fatou's Lemma, to get our result.

Remark. The reader should notice that we do not need Lemma 2.3 to prove Theorems 2.5 and 2.7: these theorems are immediate consequences of their dyadic analogues and Lemma 2.2. We have chosen to prove them this way for the sake of unity, because we will need Lemma 2.3 when we get to $\S 4$.

\section{SiNGULAR INTEGRAL OPERATORS}

Let $1<p<\infty$. In $\S 2$ we saw that

$$
\int|f|^{p} V d x \leq C(p, d) \int S_{\psi, \alpha}^{p}(f) M V d x
$$

if $1<p<2$, and

$$
\int|f|^{p} V d x \leq C(p, d, A) \int S_{\psi, \alpha}^{p}(f) M_{\rho} V d x
$$

if $2 \leq p<\infty$, for appropriate $\rho$ ( $A$ is $\rho$ 's doubling constant), $\alpha$ sufficiently large, and all $f \in \bigcup_{1<p<\infty} L^{p}\left(\mathbf{R}^{d}, d x\right)$ and weights $V$. Let $T$ be a CalderónZygmund operator as defined in the introduction. In this section we will show that, for $1<p<\infty$,

$$
\int|T f|^{p} V d x \leq C \int|f|^{p} \widetilde{M} V d x
$$

for all $f$ and $V$ as above, for appropriate "maximal functions" $\widetilde{M}$ (which depend on $p$ ). In particular, we will show that when $1<p<2$ we may take $\widetilde{M}=M^{2}$, and when $p=2$ we may take $\widetilde{M}=M^{3}$. (Unfortunately, when $2<p<\infty$, we do not know whether we are able to take $\widetilde{M}=M^{q}$ for some $q=q(p)$.

Our main tool here will be a pair of theorems which are essentially due to Chanillo and Wheeden [CW2]. In order to state them we shall need some 
notation. Let $\phi \in \mathscr{C}_{0}^{\infty}\left(\mathbf{R}^{d}\right)$ be real and radial, have support contained in $\{|x| \leq 1 / 2\}$, and satisfy $\int \phi=0$. We define

$$
g_{\lambda}^{*}(f)(x) \equiv\left(\int_{\mathbf{R}_{+}^{d+1}}\left|f * \phi_{y}(t)\right|^{2}\left(\frac{y}{y+|x-t|}\right)^{d \lambda} \frac{d t d y}{y^{d+1}}\right)^{1 / 2} .
$$

Chanillo's and Wheeden's theorems are

Theorem 3.1. Let $\lambda>3$ and $1<p \leq 2$. There is $a C=C(p, d, \lambda)$ such that

$$
\int\left(g_{\lambda}^{*}(f)\right)^{p} V d x \leq C \int|f|^{p} M V d x
$$

for all weights $V$ and all locally integrable $f$.

Theorem 3.2. Let $\lambda>1$ and $2 \leq p<\infty$. There is a $C=C(p, d, \lambda)$ such that

$$
\int\left(g_{\lambda}^{*}(f)\right)^{p} V d x \leq C \int|f|^{p} M V \cdot\left(\frac{M V}{V}\right)^{p / 2-1} d x
$$

for all $V$ and all locally integrable $f$.

Remark. Theorem 3.2 actually is proved by Chanillo and Wheeden. Theorem 3.1, while not proved (or claimed) in their paper, follows by an easy adaptation of their method (see $\S 7$ ).

The theorems we shall prove are the following:

Theorem 3.3. Let $T$ be a Calderón-Zygmund operator, and let $1<p<2$. There is a $C(p, T)$ such that

$$
\int|T f|^{p} V d x \leq C(p, T) \int|f|^{p} M(M V) d x
$$

for all weights $V$ and all $f \in \bigcup_{1<p<\infty} L^{p}\left(\mathbf{R}^{d}, d x\right)$.

Theorem 3.4. Let $T$ be a Calderón-Zygmund operator and let $2 \leq p<\infty$. Let $M_{\rho}$ be as defined in $\S 2$ (depending on $\left.p\right)$. There is a $C(p, T, A)$ such that

$$
\int|T f|^{p} V d x \leq C(p, T, A) \int|f|^{p} M\left(M_{\rho} V\right) \cdot\left(\frac{M\left(M_{\rho} V\right)}{M_{\rho} V}\right)^{p / 2-1} d x
$$

for all $V$ and all $f \in \bigcup_{1<p<\infty} L^{p}\left(\mathbf{R}^{d}, d x\right)$ ( $A$ is the doubling constant of $\rho$ ). Remark. We assume that $f \in L^{p}$ for some $p>1$ to ensure that $T f \in$ $L_{\text {loc }}^{1}\left(\mathbf{R}^{d}\right)$.

Remark. Theorem 3.3 does not hold for $p \geq 2$. First of all, it clearly fails for $p>2$, since it implies that, if $f \in L^{\infty}$, then $T f$ belongs to the exponential $L^{p / 2}$ class, which is false when $p>2$. However, we can see this more easilyand that it fails for $p=2$-with the following counterexample. Let $T \equiv$ the Hilbert transform, $V \equiv \chi_{(0,1)}$, and $f \equiv(\log x)^{-1} \chi_{\left(e, e^{n}\right)}$. A computation shows that $|T f| \geq c \log n$ on $(0,1)$, and therefore

$$
\int|T f|^{p} V d x \geq c(\log n)^{p} .
$$


But $M(M V) \sim \log (2+|x|) /(2+|x|)$; hence

$$
\int|f|^{p} M(M V) d x \leq c \int_{e}^{e^{n}} \frac{d x}{x(\log x)^{p-1}},
$$

which contradicts Theorem 3.3 as $n \rightarrow \infty$.

Let us choose $\phi$ as above, but such that $\int \phi(x) P(x) d x=0$ for every polynomial $P$ of degree $\leq 2 d$. Let $\psi \equiv \phi * \phi$. Then, after multiplication by a suitable positive constant (to get $(*)), \psi$ satisfies the hypotheses stated in the beginning of the introduction. Therefore, it will be enough to show that

$$
S_{\psi, \alpha}(T f)(x) \leq C(\alpha, \phi, \lambda) g_{\lambda}^{*}(f)(x)
$$

for all $\alpha>0$ and $f$ as above, for some $\lambda>3$. By dilation invariance, this will follow immediately from

$$
|T f * \psi(x)| \leq C(\phi, \lambda)\left(\int|f * \phi(t)|^{2}(1+|x-t|)^{-d \lambda} d t\right)^{1 / 2}
$$

But (18) is elementary. We have

$$
|T f * \psi(x)|=|f * \phi * T \phi(x)| \leq \int|f * \phi(t)||T \phi(x-t)| d t .
$$

It is easy to see that $|T \phi(x)| \leq C(T, \phi)(1+|x|)^{-3 d-1}$. Now (18) follows from an application of the Cauchy-Schwarz inequality. This proves Theorems 3.3 and 3.4.

If we choose $\phi \in \mathscr{C}^{k}\left(\mathbf{R}^{d}\right)$, and rough enough, then $\psi$ will also be rough enough, and we may obtain appropriate "grand maximal" versions of Theroems 3.3 and 3.4. We leave the statements and proofs of these theorems to the interested reader. We note in passing that these results have a nice corollary. Let us define

$$
T^{*} f(x) \equiv \sup _{\varepsilon>0}\left|\int_{|y|>\varepsilon} f(x-y) \frac{\Omega(y)}{|y|^{d}} d y\right| .
$$

It is shown in [St, pp. 67-68] that, if $f \in L^{p}, 1<p<\infty$, then

$$
T^{*} f \leq C(T)\left[G_{N}(T f)+M f\right]
$$

pointwise, where $M$ is the Hardy-Littlewood maximal function. Since we have [FS1]

$$
\int(M f)^{p} V d x \leq C(p, d) \int|f|^{p} M V d x,
$$

whenever $1<p<\infty$, we have the following corollary to the results of this section:

Theorems 3.3 and 3.4 remain true if $T f$ is replaced by $T^{*} f$.

\section{SOBOLEV INEQUALITIES}

In this section we shall assume that $1<p \leq 2$, and all the $f$ 's we deal with will belong to $\mathscr{C}_{0}^{\infty}\left(\mathbf{R}^{d}\right)$. 
We will prove two theorems, one for the case $p<2$ and the other for $p=2$.

Let us first recall a definition. We say that a weight $W$ is in the Muckenhoupt class $A_{p}(1<p<\infty)$ if

$$
\sup _{Q \subset \mathbf{R}^{d}}\left(\frac{1}{|Q|} \int_{Q} W\right)\left(\frac{1}{|Q|} \int_{Q} W^{-1 /(p-1)}\right)^{p-1} \equiv\|W\|_{A_{p}}<\infty .
$$

The value of the supremum is the $A_{p}$ "norm" of $W$ (see [M]).

Theorem 4.1. Let $1<p<2$ and let $W \in A_{p}$. Let $\eta>p / 2$. if $V$ is a weight for which

$$
l(Q)^{p} \int_{Q} V(x) \log ^{\eta}\left(e+\frac{V(x)}{V_{Q}}\right) d x \leq \int_{Q} W(x) d x
$$

for every cube $Q$ then

$$
\int|f|^{p} V d x \leq C \int|\nabla f|^{p} W d x
$$

for all $f \in \mathscr{C}_{0}^{\infty}\left(\mathbf{R}^{d}\right)$, with a constant $C$ that depends only on $\eta, p, d$, and the $A_{p}$ norm of $W$.

Theorem 4.2. Let $p=2$ and let $M_{\rho}$ be as in Theorem 2.7 (for the case $p=2$ ). Let $W \in A_{2}$. If $V$ satisfies

$$
l(Q)^{2} \int_{Q} M_{\rho}\left(\chi_{Q} V\right) d x \leq \int_{Q} W d x
$$

for every cube $Q$ then

$$
\int|f|^{2} V d x \leq C \int|\nabla f|^{2} W d x
$$

for all $f \in \mathscr{C}_{0}^{\infty}\left(\mathbf{R}^{d}\right)$. The constant $C$ depends on $d, A$ ( $\rho$ 's doubling constant), and the $A_{2}$ norm of $W$.

The only significant difference between the proofs of these two theorems is that the second one is simpler. Therefore we shall only prove Theorem 4.1.

Proof of Theorem 4.1. Let $\psi$ be as in the introduction. For $i=1, \ldots, d$, define $\phi_{i} \equiv \partial \psi / \partial x_{i}$. For $f \in \mathscr{C}_{0}^{\infty}\left(\mathbf{R}^{d}\right)$ set

$$
g_{i} \equiv \int_{\mathbf{R}_{+}^{d+1}} f *\left(\phi_{i}\right)_{y}(t)\left(\phi_{i}\right)_{y}(x-t) \frac{d t d y}{y} .
$$

By Fourier transforms, $f=c(\psi) \sum_{i} g_{i}$. The theorem will be proved if we can show, for each $i$,

$$
\int\left|g_{i}\right|^{p} V d x \leq C \int\left|\frac{\partial f}{\partial x_{i}}\right|^{p} W d x
$$

So let us now fix $i$, and set $g \equiv g_{i}$ and $\phi \equiv \phi_{i}$. Let $\mathscr{G}_{k}$ and $g_{(k)}$ be the families and functions obtained via Lemma 2.1. For each $k$ we have

$$
g_{(k)}=\sum_{Q \in \mathscr{F}_{k}} \lambda_{Q} a_{Q}
$$


where we may set, for $\widetilde{Q} \in \mathscr{G}_{k}$,

$$
\left|\lambda_{\widetilde{Q}}\right| \leq C\left(\int_{T(Q)}\left|f * \phi_{y}(t)\right|^{2} \frac{d t d y}{y}\right)^{1 / 2}
$$

We shall for the moment fix $k$ and set $g \equiv g_{(k)}$. Let $\mathscr{F}_{j}, g_{j}$, and $c_{Q}\left(g_{j}\right)$ be as defined in the proof of Theorem 2.5. The argument given there yields

$$
\int|g|^{p} V d x \leq C(p, d, \eta) \sum_{j}(1+j)^{2} 2^{j(p / 2 \eta-1)} \sum_{Q \in \mathscr{F}_{j}} c_{Q} l(Q)^{-p} W(Q) .
$$

Now, since $p / 2<1$,

$$
\begin{aligned}
c_{Q} l(Q)^{-p} & =\left(\sum_{Q \subseteq Q^{\prime} \in \mathscr{F}_{j}} \frac{\left|\lambda_{Q^{\prime}}\right|^{2} l(Q)^{-2}}{\left|Q^{\prime}\right|}\right)^{p / 2}-\left(\sum_{\substack{Q \subset Q^{\prime} \in \mathscr{F}_{j} \\
Q \neq Q^{\prime}}} \frac{\left|\lambda_{Q^{\prime}}\right|^{2} l(Q)^{-2}}{\left|Q^{\prime}\right|}\right)^{p / 2} \\
& \leq\left(\sum_{Q \subseteq Q^{\prime} \in \mathscr{F}_{j}} \frac{\left|\lambda_{Q^{\prime}}\right|^{2} l\left(Q^{\prime}\right)^{-2}}{\left|Q^{\prime}\right|}\right)^{p / 2}-\left(\sum_{\substack{Q \subset Q^{\prime} \in \mathscr{F}_{j} \\
Q \neq Q^{\prime}}} \frac{\left|\lambda_{Q^{\prime}}\right|^{2} l\left(Q^{\prime}\right)^{-2}}{\left|Q^{\prime}\right|}\right)^{p / 2} .
\end{aligned}
$$

(Here we are using the fact that $(x+a)^{\alpha}-x^{\alpha} \leq(y+a)^{\alpha}-y^{\alpha}$ if $x, y$, and $a$ are nonnegative, $x \geq y$, and $0<\alpha \leq 1$. In our case,

$$
\begin{aligned}
& \alpha=\frac{p}{2}, \quad x=\sum_{\substack{Q \subset Q^{\prime} \subset \mathscr{F}_{j} \\
Q \neq Q^{\prime}}} \frac{\left|\lambda_{Q^{\prime}}\right|^{2} l(Q)^{-2}}{\left|Q^{\prime}\right|}, \\
& y=\sum_{\substack{Q \subset Q^{\prime} \in \mathcal{F}_{j} \\
Q \neq Q^{\prime}}} \frac{\left|\lambda_{Q^{\prime}}\right|^{2} l\left(Q^{\prime}\right)^{-2}}{\left|Q^{\prime}\right|}, \quad a=\frac{\left|\lambda_{Q}\right|^{2} l(Q)^{-2}}{|Q|} .
\end{aligned}
$$

We get equality if $p=2$.)

Set $\gamma_{Q} \equiv \lambda_{Q} \cdot l(Q)^{-1}$. Summing on $k$ and $j$ yields, for each $i$, (17)

$$
\begin{aligned}
\int\left|g_{i}\right|^{p} V d x & \leq C(p, d, \eta) \int\left(\sum_{x \in Q \in \cup \mathscr{G}_{k}} \frac{\left|\gamma_{Q}\right|^{2}}{|Q|}\right)^{p / 2} W d x \\
& \leq C(p, d, \eta) \int\left(\int_{|x-t|<3 \sqrt{d y}}\left|f *\left(\phi_{i}\right)_{y}(t) y^{-1}\right|^{2} \frac{d t d y}{y^{d+1}}\right)^{p / 2} W d x .
\end{aligned}
$$

But $f *\left(\phi_{i}\right)_{y}(t) y^{-1}=\partial t / \partial x_{i} * \psi_{y}(t)$. Therefore the right-hand side of (17) equals a constant times

$$
\int S_{\psi, 3 \sqrt{d}}^{p}\left(\frac{\partial f}{\partial x_{i}}\right) W d x
$$


and it is well-known $[R F, K]$ that this is less than or equal to

$$
C \int\left|\frac{\partial f}{\partial x_{i}}\right|^{p} W d x
$$

when $W \in A_{p}$. Q.E.D.

We wish to make an observation about Theorem 4.2 which will be useful in the following section. Let $\rho$ be as in the theorem. We claim that the conclusion of the theorem holds if $W \in A_{2}$ and if, for all cubes $Q$,

$$
l(Q)^{2} \int_{Q} V(x) \log \left(e+\frac{V(x)}{V_{Q}}\right) \rho\left(\log \log \left(10+\frac{V(x)}{V_{Q}}\right)\right) d x \leq \int_{Q} W d x .
$$

The reason for this is that we only need for the "dyadic" version of (16) to hold, for each family $\mathscr{G}_{k}$. But the argument from [W2] shows that

$$
\int_{Q} M_{\rho}\left(\chi_{Q} V\right) d x \leq C \int_{Q} V(x) \log \left(e+\frac{V(x)}{V_{q}}\right) \rho\left(\log \log \left(10+\frac{V(x)}{V_{Q}}\right)\right) d x
$$

is true in that case. This proves our assertion.

\section{SCHRÖDINGER OPERATORS}

In this section we shall assume that $d>2$. We shall also assume that $\psi$ (see the introduction) satisfies $\int \psi(x) P(x) d x=0$ for all polynomials $P$ with degree $\leq 1$, and that $\psi \in \mathscr{C}^{k}\left(\mathbf{R}^{d}\right)$ for $k \geq 3$. It is easy to see that the adapted functions $b_{Q}$ defined via this $\psi$ inherit $\psi$ 's cancellation property and also satisfy $\left\|D^{\alpha} b_{Q}\right\|_{\infty} \leq C|Q|^{-1 / 2} l(Q)^{-2}$ for all multi-indices $|\alpha|=2$.

Let $A(x)=\left(a_{i j}(x)\right)$ be a $d \times d$ real matrix-valued function of $x \in \mathbf{R}^{d}$. We assume that $A(x)$ is symmetric and positive semidefinite, with eigenvalues $\lambda_{1}(x) \leq \cdots \leq \lambda_{d}(x)$. We assume furthermore that there exist positive constants $c_{1}$ and $c_{2}$, and an $A_{2}$ weight $W$ such that

$$
c_{1} W(x) \leq \lambda_{1} \leq \lambda_{d} \leq c_{2} W(x)
$$

for all $x \in \mathbf{R}^{d}$. For $V$ nonnegative in $L_{\text {loc }}^{1}\left(\mathbf{R}^{d}\right)$, we consider the Schrd̈inger operator $L$, defined by

$$
L f \equiv-\operatorname{div}(A(x) \nabla f)-V \cdot f .
$$

We assume that $V$ and $W$ are sufficiently regular so that everything we say about $L$ makes sense.

Such operators have recieved a great deal of attention recently. In particular, the case where $A \equiv I$, the identity, was studied in [F, CWW, and $\mathrm{KS}]$; while the case of nonconstant $A$ was treated in [CW1]. We shall describe some of these results and show their relation to our own. Since [F] was the earliest of these papers, and it established the basic procedure which the other researchers have followed, our discussion of $[F]$ will be the most complete. 
In studying the spectrum of operators like $L$, the central question is: When is $L$ nonnegative? In the case when $A$ is the identity matrix, integration by parts shows that $L$ 's nonnegativity is equivalent to

$$
\int|f|^{2} V d x \leq \int|\nabla f|^{2} d x
$$

for all $f \in \mathscr{C}_{0}^{\infty}\left(\mathbf{R}^{d}\right)$. In [F], the following is shown; given $p>1$ there is a $\gamma(p, d)>0$ so that if, for all cubes $Q$,

$$
l(Q)^{2}\left(\frac{1}{|Q|} \int_{Q} V^{p} d x\right)^{1 / p} \leq \gamma(p, d)
$$

then $L$ is nonnegative. Using this result, one is able to get rather precise estimates of the size of $L$ 's lowest (negative) eigenvalue, in the case when $L$ is not nonnegative. Call this eigenvalue $\lambda_{1}(L)$. One has

$$
c_{1} \sup _{Q}\left[V_{Q}-c_{2} l(Q)^{-2}\right] \leq-\lambda_{1}(L) \leq c_{3} \sup _{Q}\left[\left(\frac{1}{|Q|} \int_{Q} V^{p} d x\right)^{1 / p}-c_{4} l(Q)^{-2}\right]
$$

where the positive constants $c_{i}$ depend only on $p$ and $d$.

One can also count the negative eigenvalues of $L$, in the following sense. Let $\lambda>0$. If there exist cubes $Q_{1}, \ldots, Q_{N}$ with disjoint doubles such that, for each $i, V_{Q_{i}}>c_{1} l\left(Q_{i}\right)^{-2}, l\left(Q_{i}\right) \leq c_{2} \lambda^{-1 / 2}$, where the $c_{i}>0$ depend on $d$, then $L$ has at least $N$ eigenvalues less then or equal to $-\lambda$. And: if $L$ has $N$ negative eigenvalues, and $p>1$, then there exist $c_{1} N$ disjoint cubes $Q_{i}$ such that

$$
\left(\frac{1}{\left|Q_{i}\right|} \int_{Q_{i}} V^{p} d x\right)^{1 / p} \geq c_{2} l\left(Q_{i}\right)^{-2}, \quad l\left(Q_{i}\right) \leq c_{3} \lambda^{-1 / 2},
$$

for each $i$, where, again, the $c_{j}$ are positive constants that depend on $p$ and $d$.

In order to sharpen these resutls, one first of all wants to find something strictly weaker than $(21)$ which will imply $L \geq 0$. One such condition is given in [CWW]. Let $\phi:[0, \infty) \rightarrow[1, \infty)$ be increasing and satisfy $\phi(4 x) \leq A \phi(x)$ for all $x$, for some $A \leq 2^{d-2}$. In [CWW] it is shown that if

$$
\int_{1}^{\infty} \frac{d x}{x \phi(x)} \leq 1
$$

and

$$
\sup _{Q} l(Q)^{2} \int_{Q} V(x) \phi\left(l(Q)^{2} V(x)\right) d x \leq c(d, A)
$$

then $L \geq 0$. At first sight, (20) may not seem to be the right extension of (21), since the homogeneity appears to be wrong. The right extension, one thinks, should be

$$
\sup _{Q} l(Q)^{2} \int_{Q} V(x) \phi\left(\frac{V(x)}{V_{Q}}\right) d x \leq c^{\prime}(d, A) .
$$


But, in fact, (23) implies (22), since (23) implies that $V_{Q} \leq c^{\prime}(d, A) l(Q)^{-2}$. By means of the same procedure as that in $[F]$, the analogous eigenvalue estimates now follow.

In [KS], Kerman and Sawyer found (modulo a positive multiplicative constant) a highly nontrivial necessary and sufficient condition on $V$ for $L$ to be nonnegative. Define

$$
M_{1} V(x) \equiv \sup _{x \in Q} \frac{l(Q)}{|Q|} \int_{Q} V d t
$$

Their result is the following: There is a $C_{1}<\infty$ such that

$$
\int|f|^{2} V d x \leq C_{1} \int|\nabla f|^{2} d x
$$

for all test functions $f$, if and only if there is a $C_{2}<\infty$ such that

$$
\int_{Q}\left[M_{1}\left(\chi_{Q} V\right)\right]^{2} d x \leq C_{2} \int_{Q} V d x
$$

for all cubes $Q$, where $C_{1} / C_{2}$ is bounded above and below by positive constants that depend only on the dimension. They obtain results, analogous to those of [F], in which $\int_{Q}\left[M_{1}\left(\chi_{Q} V\right)\right]^{2} / \int_{Q} V$ plays the role of $l(Q)^{2}\left(\frac{1}{|Q|} \int_{Q} V^{p}\right)^{1 / p} .^{1}$

Now let $L$ be as in the first paragraph of this section, but with $A \not \equiv I$. Integration by parts shows that $L \geq 0$ follows from the inequality

$$
\int|f|^{2} V d x \leq c \int|\nabla f|^{2} W d x
$$

for all test functions $f$, for $c>0$ sufficiently small. The methods of [KS] show that (24) holds if and only if

$$
\int_{Q}\left[M_{1}\left(\chi_{Q} V\right)\right]^{2} W^{-1} d x \leq C \int_{Q} V d x
$$

for all cubes $Q$. This is because, if $W \in A_{2},(24)$ is equivalent to an inequality involving Riesz potentials, to which the methods of [KS] apply.

Unfortunately, although $(25)$ is an inequality that only needs to be tested over cubes, if $V$ is not simple, this can still be rather difficult to verify, even if $W \equiv 1$. Therefore it is desirable to find a condition on $V$ and $W$ which, while less sharp than (25), looks more like those in [CWW and F], and is therefore easier to check.

One way to do this is to apply the method of [CWW] directly to the weighted case. This has been done by Chanillo and Wheeden [CW1]. Let $\phi:[0, \infty) \rightarrow$ $[1, \infty)$ be increasing. Suppose that $\phi$ satisfies

$$
\int_{1}^{\infty} \frac{d t}{t \phi(t)}<\infty
$$

1 The Fefferman-Phong and Kerman-Sawyer results have also been obtained, through different means, by Schechter [Sch]. 
Chanillo and Wheeden show that if $V$ and $W$ are weights such that

$$
l(Q)^{2} \int_{Q} \phi\left(\frac{l(Q)^{2} V(x)}{W(x)}\right) V(x) d x \leq \int_{Q} W(x) d x
$$

for all cubes $Q$ then (24) holds, with a constant $c$ that depends on $\phi, d, W$ 's $A_{2}$ norm, and the constants in (19). By choosing $\phi=(1+t)^{\varepsilon}$ they are able to show that (24) follows if

$$
l(Q)^{2}\left(\frac{1}{|Q|} \int_{Q} V^{p}\right)^{1 / p} \leq C(p, d) W_{Q}
$$

for all cubes $Q$, for any $p>1$. This is the right generalization of the FeffermanPhong condition; in particular, it has the correct homogeneity. Following [F], Chanillo and Wheeden are able to get the appropriate eigenvalue estimates for $L$. However, there does not seem any way to transform (27), for general $\phi$, into the appropriate homogeneous form.

The other approach to this problem is to derive (24) from a two-weight square function inequality, and this is what we have done. Let $\rho:[0, \infty) \rightarrow[1, \infty)$ be increasing and satisfy $\rho(2 x) \leq A \rho(x)$ for all $x$. Furthermore assume that $\sum 1 / \rho(k) \leq 1$. In the previous section it was shown that if

$$
l(Q)^{2} \int_{Q} V(x) \log \left(e+\frac{V(x)}{V_{Q}}\right) \rho\left(\log \log \left(10+\frac{V(x)}{V_{Q}}\right)\right) d x \leq \int_{Q} W(x) d x
$$

for all cubes $Q$, then (24) holds with a constant $c$ that depends on $A, d$, the $A_{2}$ norm of $W$, and the constants in (19). It should be noticed that $\phi \equiv \log (e=x) \rho(\log \log (10+x))$ satisfies (26). Therefore (28) is the appropriate homogeneous form of the Chanillo-Wheeden result, and is thus the appropriate weighted form of the result from [CWW].

Let us know fix $\rho$ for the rest of this section, and define

$$
\Lambda(Q, V) \equiv \int_{Q} V(x) \log \left(e+\frac{V(x)}{V_{Q}}\right) \rho\left(\log \log \left(10+\frac{V(x)}{V_{Q}}\right)\right) d x
$$

We are now ready to state our theorems ( $A$ will henceforth refer to $\rho$ 's doubling constant).

Theorem 5.1. Let $A(x)$ be as in the first paragraph of this section, with $W \in A_{2}$. let $L \equiv-\operatorname{div}(A(x) \cdot)-V(x) \cdot$, and let $\lambda_{1}(L)$ be $L$ 's lowest nonpositive eigenvalue. There are constants $c_{1}, c_{2}, c_{3}, c_{4}$ which depend on $A, d$, the constants in (19), and $W$ 's $A_{2}$ norm, such that

$$
\sup _{Q} c_{1}\left[V_{Q}-c_{2} l(Q)^{-2} W_{Q}\right] \leq-\lambda_{1}(L) \leq \sup _{Q} c_{3}\left[\frac{1}{|Q|} \Lambda(Q, V)-c_{4} l(Q)^{-2} W_{Q}\right] \text {. }
$$

Remark. The upper bound in (29) is the analogue to an estimate obtained in [CW1, Theorem 1.10]. However, it is assume there that $W \in D_{\mu}$ for $\mu<$ 
$1+2 / d$, where we say that $W \in D_{\beta}$ if there is a finite $C$ so that

$$
W(Q) \leq C\left(|Q| /\left|Q^{\prime}\right|\right)^{\beta} W\left(Q^{\prime}\right)
$$

for all cubes $Q \supset Q^{\prime}$. Since we are assuming $W \in A_{2}$, we have $W \in D_{\beta}$ for some $\beta>0$. But our result holds without assuming an upper bound on $\beta$.

In order to apply the Fefferman-Phong recipe to $L$ we shall need to assume, at one point, an additional hypothesis on $W$. Let us say that $W \in R D_{\beta}$ (reverse doubling of order $\beta>0$ ) if there is a finite $C$ so that for all cubes $Q \subset Q^{\prime}$,

$$
W(Q) \leq C\left(|Q| /\left|Q^{\prime}\right|\right)^{\beta} W\left(Q^{\prime}\right) .
$$

Theorem 5.2. Let $L$ be as above. There are constants $c_{1}, c_{2}, c_{3}$, depending on $A, d$, the constants in (19), and $W$ 's $A_{2}$ norm, such that the following statements hold:

(i) If there are cubes $Q_{1}, \ldots, Q_{N}$, with disjoint doubles such that

$$
l\left(Q_{i}\right)^{2} V\left(Q_{i}\right) \geq c_{1} W\left(Q_{i}\right)
$$

for each $i$, then $L$ has at least $N$ negative eigenvalues;

(ii) Assume that, in addition, $W \in R D_{\beta}$ for some $\beta>2 / d$. If $L$ has at least $N<\infty$ negative eigenvalues, then there are disjoint cubes $Q_{1}, \ldots, Q_{M}$, where $M \geq c_{2} N$, such that

$$
l\left(Q_{j}\right)^{2} \Lambda\left(Q_{j}, V\right) \geq c_{3} W\left(Q_{j}\right)
$$

for each $j$.

Proof of Theorem 5.1. We will only prove the right-hand inequality in (29) (the left-hand one is elementary: see [F, CW1]). Assume that, for all cubes $Q$,

$$
\frac{1}{|Q|} \Lambda(Q, V)-c_{4} l(Q)^{-2} W_{Q} \leq B
$$

where $c_{4}$ will be chosen later. We will show that $L \geq-c B$, for some positive constant $c$.

Inequality (30) implies that

$$
\Lambda(Q, V) \leq c_{4} l(Q)^{-2} W(Q)+B|Q|
$$

for all $Q$. Now take $f \in \mathscr{C}_{0}^{\infty}\left(\mathbf{R}^{d}\right)$ and write $f=c(\psi) \sum_{i, k}\left(g_{i}\right)_{(k)}$, where the $\left(g_{i}\right)_{(k)}$ are as in the proof of Theorem 4.1. For each $\left(g_{i}\right)_{(k)}$ we have

$$
\begin{aligned}
\int\left|\left(g_{i}\right)_{(k)}\right|^{2} V d x & \leq C \sum_{Q \in \mathscr{G}_{k}} \frac{\left|\lambda_{Q}\right|^{2}}{|Q|} \Lambda(Q, V) \\
& \leq C \sum_{Q \in \mathscr{G}_{k}} \frac{\left|\lambda_{Q}\right|^{2}}{|Q|}\left[c_{4} l(Q)^{-2} W(Q)+B|Q|\right] \\
& \leq C c_{4} \int|\nabla f|^{2} W d x+c B \int|f|^{2} d x,
\end{aligned}
$$


where (31) follows from the arguments in $\S 4$ and the fact that the square function is bounded on $L^{2}\left(\mathbf{R}^{d}, d x\right)$. Summing on $i$ and $k$ implies

$$
\int|f|^{2} V d x \leq C c_{4} \int|\nabla f|^{2} W d x+c B \int|f|^{2} d x .
$$

Thus, if $c_{4}$ is small enough, we get $L \geq-c B$. Q.E.D.

Proof of Theorem 5.2. Since (i) is obvious (see [F]), we will only prove (ii). We shall first assume, much as in [F], that $V \leq R W$, for some $R>0$, and that $V$ has compact support; the bounds we get will not depend on $R$ or the size of the support; at the end we will show how to remove this restriction. For $1 \leq k \leq 3^{d}$ let $\left\{Q_{j}^{k}\right\}$ be the minimal $Q \in \mathscr{G}_{k}$ such that $\Lambda(Q, V) \geq c_{3} l(Q)^{-2} W(Q)$, where $c_{3}>0$ is to be determined. ${ }^{2}$ Let $n_{k}$ be the number of such cubes. The cubes in the conclusion of the theorem will be the minimal $Q \in \mathscr{G}_{j}$ for which $n_{j}$ is largest; we will show that $L$ has at most $C \sum n_{k}$ negative eigenvalues.

Let us temporarily fix $k$. Following the procedure described in [F], we pick additional cubes $\left\{Q_{(j)}^{k}\right\} \subset \mathscr{G}_{k}$. We can do this because the cubes in $\mathscr{G}_{k}$ have the same inclusion properties as the dyadic cubes. It is shown in [F] that we get no more than $C n_{k}$ additional cubes. Take the union of $\left\{Q_{j}^{k}\right\}$ and $\left\{Q_{(j)}^{k}\right\}$, and call resulting set $\left\{Q_{j}^{k}\right\}$. For $1 \leq i \leq d$ and $1 \leq k \leq 3^{d}$ let us defined bounded linear operators $l_{i, k}: L^{2}\left(\mathbf{R}^{d}, d x\right) \rightarrow L^{2}\left(\mathbf{R}^{d}, d x\right)$ by $l_{i, k}(f) \equiv\left(g_{i}\right)_{(k)}$, where the $\left(g_{i}\right)_{(k)}$ are as in the proof of Theorem 4.1. We define a closed subspace $H \subset L^{2}$ by saying that $f \in H$ if, for each $i$ and $k$,

$$
\int_{Q} l_{i, k}(f) P(x) d x=0 \quad \forall Q \in\left\{Q_{j}^{k}\right\},
$$

for all polynomials $P$ of degree $\leq 1$. Clearly $H$ has codimension $\leq c \sum n_{k}$. We will be done once we show that

$$
\int|f|^{2} V d x \leq c \int|\nabla f|^{2} W d x
$$

for all $f \in H \cap \mathscr{C}_{0}^{\infty}\left(\mathbf{R}^{d}\right)$, for sufficiently small $c$. So let us take such an $f$ and write $f=c(\psi) \sum\left(g_{i}\right)_{(k)}$, where each $\left(g_{i}\right)_{(k)}$ satisfies (33).

We shall follow the arguments in [F and CW1]. Define $Q_{0}^{k} \equiv \mathbf{R}^{d}$. For each $1 \leq k \leq 3^{d}$, let $\mathscr{B}_{k}$ be those $Q \in \mathscr{G}_{k}$ such that $\Lambda(Q, V) \geq c_{3} l(Q)^{-2} W(Q)$. For each $Q \in\left\{Q_{j}^{k}\right\}$ define

$$
E(Q) \equiv Q \backslash \bigcup_{\substack{j: Q_{j}^{k} \subset Q \\ Q_{j}^{k} \neq Q}} Q_{j}^{k},
$$

${ }^{2}$ These minimal cubes exist if $V \leq R W$, since $W \in A_{\infty}$ implies $\Lambda(Q, V) \leq C_{R} \Lambda(Q, W) \leq$ $C \cdot C_{R} W(Q)$. Similarly, the analogous maximal cubes exist because $W \in R D_{\beta} \quad(\beta>2 / d)$ and $V$ has compact support. 
and set

$$
E\left(Q_{0}^{k}\right) \equiv \mathbf{R}^{d} \backslash \bigcup_{j>0} Q_{j}^{k} .
$$

We will have (34) once we show the following two statements:

(i) If $Q \in \mathscr{G}_{k}$ and $Q \not \subset Q_{j}^{k}$ for any $j$ then $\Lambda(Q, V) \leq C \cdot c_{3} l(Q)^{-2} W(Q)$ (note: we shall call this family of cubes $\mathscr{R}_{0}$ ).

(ii) If $Q \in \mathscr{G}_{k}$ and $Q \subset Q_{j}^{k}$ for some $j$, but $Q \not \subset Q_{j^{\prime}}^{k}$ for any $Q_{j^{\prime}}^{k}$ strictly contained in $Q_{j}^{k}$, then $\Lambda\left(Q, V \chi_{E\left(Q_{j}^{k}\right)}\right) \leq C \cdot c_{3} l(Q)^{-2} W(Q)$ (note: we shall call this family of cubes $\mathscr{R}_{j}$ ).

We shall first show that (i) and (ii) imply (34). Let us set $Q_{0}^{k} \equiv \mathbf{R}^{d}$, and fix $g \equiv\left(g_{i}\right)_{(k)}$. For $Q \in \mathscr{G}_{k}$, let $\tilde{a}_{Q}(g)$ be the corresponding "dyadic" martingale difference as defined in the remark following the proof of Lemma 2.2. Let $\widetilde{S}(\cdot)$ denote the corresponding dyadic square function. Set, for $j \geq 0$,

$$
g_{j} \equiv \sum_{Q \in \mathscr{R}_{j}} \tilde{a}_{Q}(g)
$$

Since $g$ satisfies (33), we have $g \equiv g_{j}$ on $E\left(Q_{j}^{k}\right)$, for all $j$. Also, the $E\left(Q_{j}^{k}\right)$ 's make a partition of $\mathbf{R}^{d}$. Therefore,

$$
\begin{aligned}
& \int|g|^{2} V d x=\sum_{j} \int_{E\left(Q_{j}^{k}\right)}\left|g_{j}\right|^{2} V d x \\
& \leq C \sum_{j} \sum_{Q \in \Re_{j}} \frac{\left\|\tilde{a}_{Q}(g)\right\|_{2}^{2}}{|Q|} \Lambda\left(Q, V \chi_{E\left(Q_{j}^{k}\right)}\right) \\
& \leq C \cdot c_{3} \sum_{j} \sum_{Q \in \Re_{j}} \frac{\left\|\tilde{a}_{Q}(g)\right\|_{2}^{2}}{|Q|} l(Q)^{-2} W(Q) \\
& \leq C \cdot c_{3} \int \sum_{x \in Q} \frac{\left\|\tilde{a}_{Q}(g)\right\|_{2}^{2}}{|Q|} l(Q)^{-2} W d x \\
& \leq C \cdot c_{3} \int \sum_{x \in Q} \frac{|\lambda|^{2}}{|Q|} l(Q)^{-2} W d x \\
& \leq C \cdot c_{3} \int S_{\psi, \alpha}^{2}\left(\frac{\partial f}{\partial x_{i}}\right) W d x \\
& \leq C \cdot c_{3} \int\left|\frac{\partial f}{\partial x_{i}}\right|^{2} W d x,
\end{aligned}
$$

where (35) and (37) are from the remark following the proof of Lemma 2.2 ((35) holds because the same good- $\lambda$ inequality argument works for $\widetilde{S}$ as for $S$; see [W2]), (36) is from (i) and (ii), and the last line is because $W \in A_{2}$. Therefore we only need to prove (i) and (ii). 
Our argument follows that in [F] almost verbatim. We can assusme that $Q \in \mathscr{B}_{k}$ and, because of Lemma 5.3 and the argument in [F], we only need to check the case where $Q \in \mathscr{R}_{j}, Q \neq Q_{j}^{k}$, and $Q_{j}^{k}$ is (in the sense of [F]) not branching. We let $Q^{\#} \in \mathscr{G}_{k}$ be the (unique) maximal subcube of $Q_{j}^{k}$ which is either branching or minimal. As in [F], if $Q \in \mathscr{B}_{k}$ and $Q \cap E\left(Q_{j}^{k}\right) \neq \varnothing$, then $Q^{\#} \subset Q$ and $Q^{\#} \neq Q$. Following [F], we are able to write $Q \backslash Q^{\#}$ as a disjoint union of cubes $Q_{\alpha}$ satisfying $\Lambda\left(Q_{\alpha}, V\right) \leq c_{3} l\left(Q_{\alpha}\right)^{-2} W\left(Q_{\alpha}\right)$. For each positive integer $n$, there are no more than $2^{d}-1 Q_{\alpha}$ 's with sidelength $2^{-n} l(Q)$. Each of these cubes will satisfy $V_{Q_{n}} \leq 2^{n d}\left(V \chi_{E\left(Q_{j}^{k}\right)}\right)_{Q}$. Finally, let us define $\pi(x) \equiv \log (e+x) \rho(\log \log (10+x))$, and observe that there is a $C$ such that $\pi(a b) \leq C(\pi(a)+\pi(b))$ for all nonnegative $a$ and $b$. Therefore,

$$
\begin{aligned}
\Lambda\left(Q, V \chi_{E\left(Q_{j}^{k}\right)}\right) & \leq C \sum_{\alpha}\left[\int_{Q_{\alpha}} V(x) \pi\left(\frac{V(x)}{V_{Q_{\alpha}}}\right) d x+\int_{Q_{\alpha}} V(x) \pi\left(\frac{V_{Q_{\alpha}}}{\left(V \chi_{E\left(Q_{j}^{k}\right)}\right)_{Q}}\right) d x\right] \\
& \leq C \cdot c_{3} \sum_{\alpha} l\left(Q_{\alpha}\right)^{-2} W\left(Q_{\alpha}\right)+C \sum_{n=1}^{\infty} n^{2} \sum_{l\left(Q_{n}\right)=2^{-n} l(Q)} V\left(Q_{\alpha}\right) . \\
& \leq C \cdot c_{3} \sum_{n=1}^{\infty} \sum_{l\left(Q_{n}\right)=2^{-n} l(Q)} l\left(Q_{\alpha}\right)^{-2} W\left(Q_{\alpha}\right)\left[1+n^{2}\right] \\
& \leq C \cdot c_{c} \cdot\left(2^{d}-1\right) l(Q)^{-2} W(Q) \sum_{n=1}^{\infty} 2^{2 n} 2^{-d \beta n}\left[1+n^{2}\right] \\
& \leq C \cdot c_{3} l(Q)^{-2} W(Q),
\end{aligned}
$$

where (38) is because $W \in R D_{\beta}$ and (39) is because $\beta>2 / d$. This proves Theorem 5.2 when $V / W \in L^{\infty}$ and $V$ has compact support.

Let us now show how to remove our restriction on $V$. For $R>0$ define

$$
V^{R}(x) \equiv \begin{cases}V(x) & \text { if } V(x) \leq R W(x) \text { and }|x| \leq R \\ R W(x) & \text { if } V(x)>R W(x) \text { and }|x| \leq R \\ 0 & \text { if }|x|>R\end{cases}
$$

Define $L^{R} \equiv-\operatorname{div}(A(x) \nabla \cdot)-V^{R}$. Assume that $L$ has at least $N$ negative eigenvalues, i.e., that there exist orthonormal $\phi_{1}, \ldots, \phi_{N} \in \mathscr{C}_{0}^{\infty}\left(\mathbf{R}^{d}\right)$ such that $\left(L \phi_{i}, \phi_{i}\right)<0$ for each $i$. Then, for $R$ sufficiently large, $L^{R}$ will also have $N$ negative eigenvalues, and the minimal cubes obtained for $V^{R}$ will satisfy $\Lambda(Q, V) \geq c_{3} l(Q)^{-2} W(Q)$, with possibly a smaller $c_{3}$. This finishes the proof of Theorem 5.2. Q.E.D.

Remark. Using the same argument as above, one can prove the following theorem:

Theorem 5.3. Let $V, W$, and $L$ be as in Theorem 5.2. Let $E>0$. 
(i) If there exist cubes $Q_{1}, \ldots, Q_{N}$ with disjoint doubles, such that

$$
V_{Q_{i}}-c_{1} l\left(Q_{i}\right)^{-2} W_{Q_{i}} \geq c_{2} E
$$

for each $i$, then $L$ has at least $N$ eigenvalues $<-E$.

(ii) Assume that $W \in R D_{\beta}$ for some $\beta<2 / d$. If $L$ has at least $N<\infty$ eigenvalues $<-E$, then there exist disjoint cubes $Q_{1}, \ldots, Q_{M}$, with $M \geq c_{3} N$, such that

$$
\frac{1}{\left|Q_{i}\right|} \Lambda\left(Q_{i}, V\right)-c_{4} l\left(Q_{i}\right)^{-2} W_{Q_{i}} \geq c_{5} E
$$

for each $i$, where the positive constants $c_{1}, \ldots, c_{5}$ depend on the usual parameters.

Remark. The $c_{4}$ in Theorem 5.3 can be taken to equal the $c_{3}$ in Theorem 5.2.

Proof of Theorem 5.3. Again, (i) is almost obvious, and we will not prove it.

We prove (ii) almost exactly as in Theorem 5.2. The only difference is that instead of looking for the minimal $Q \in \mathscr{G}_{k}$ which have

$$
\Lambda(Q, V) \geq c_{3} l(Q)^{-2} W(Q),
$$

we look for the minimal $Q$ which satisfy (40). Details are left to the reader. Q.E.D.

Remark. Theorem 5.3(ii) does not quite have the same form as its analogues in [F and $\mathrm{CW} 1$ ]. The appropriate analogue should be

Let $L$ have $N$ eigenvalues $<-E$. Let $B$ be those minimal cubes obtained in Theorem 5.2. Then there exist $Q_{1}, \ldots, Q_{M}$, with $M \geq c_{1} N$, belonging to $B$, such that

for each $i$.

$$
l\left(Q_{i}\right)^{-2} W_{Q_{1}} \geq c_{2} E
$$

We have stated our results the way we have because in order to prove the preceding statement, we need to assume an additional hypothesis on $W$, such as that $W \in D_{\mu}$ for some $\mu<1+2 / d$. The reason for this is that, in the two-weight case, the place of the cubes with sidelength $\sim E^{-1 / 2}$ is taken by the maximal "dyadic" cubes for which $l(Q)^{-2} W_{Q} \geq c E$, and if we do not make some such hypothesis on $W$, we do not know that such maximal cubes exist (see [CW1]).

\section{Sмоотн $\psi$ 's}

In this section we sketch how to prove (the analogues of) the square functions results from $\S 2$ in the case when $\psi \in \mathscr{C}_{0}^{\infty}\left(\mathbf{R}^{d}\right)$.

Let $H$ be as defined in $\S 2$. In this case we do not have the straightforward estimate of Lemma 2.8. However, we have replacement. For $\lambda>1$ define

$$
H_{\lambda}^{* *}(f)(x) \equiv \sup _{(t, y) \in \mathbf{R}_{+}^{d+1}}\left|f * H_{y}(t)\right|\left(\frac{y}{y+|x-t|}\right)^{d \lambda} .
$$


This is the "tangential" maximal function introduced by Fefferman and Stein in [FS2].

We have the following theorem [FS2]:

Theorem 6.1. For every $\lambda>0$ there is an $N=N(H, \lambda)$ such that

$$
G_{N}(f)(x) \leq C H_{\lambda}^{* *}(f)(x)
$$

for all $f \in L_{\mathrm{loc}}^{1}\left(\mathbf{R}^{d}\right)$ and all $x \in \mathbf{R}^{d}$. The constant $C$ depends on $H, N$, and $\lambda$.

Therefore we will have our grand maximal theorems once we find some way to control $H_{\lambda}^{* *}$. Let us set $H^{+}(f)(x) \equiv \sup _{y>0}\left|f * H_{y}(x)\right|$; we already know how to control $H^{+}$.

Now, it is easy to see that

$$
H_{\lambda}^{* *}(f)(x) \leq C\left(H^{+}(f)(x)+\sum_{k=0}^{\infty} 2^{-k d \lambda} \sup _{\substack{y>0 \\|x-t|<2^{k} y}}\left|f * H_{y}(x)-f * H_{y}(t)\right|\right) .
$$

But each term in the summation is less than or equal to $C k 2^{-k d \lambda} S_{\psi, b \cdot 2^{k}}(f)$. This clearly implies that

$$
H_{\lambda}^{* *}(f)(x) \leq C\left(H^{+}(f)(x)+g_{\lambda^{\prime}}^{*}(f)(x)\right)
$$

for $\lambda^{\prime}>\lambda-\varepsilon$, with $\varepsilon>0$ as small as we please. Therefore,

$$
G_{N}(f)(x) \leq C\left(H^{+}(f)(x)+g_{\lambda}^{*}(f)(x)\right) .
$$

Since is is obvious that $S_{\psi, \alpha}(f) \leq C g_{\lambda}^{*}(f)$, we can now state the appropriate analogue of our results from $\S 2$ :

Theorem 6.2. For $0<p<\infty$ let $V$ and $W$ satisfy the hypotheses of one of the theorems from $\S 2$. Let $\lambda>1$. There is a finite $C$ so that

$$
\int\left|G_{N}(f)\right|^{p} V d x \leq C \int\left(g_{\lambda}^{*}(f)\right)^{p} W d x
$$

for all $f$ in the appropriate test class (depending on $p$ ).

\section{APPENDiX: the Chanillo-WheEden INEQUality}

We wish to supply the small argument needed to prove Theorem 3.1.

Since $g_{\lambda^{\prime}}^{*}(f) \leq g_{\lambda}^{*}(f)$ whenever $\lambda^{\prime} \geq \lambda$, it is sufficient to prove the theorem for $3<\lambda<4$.

Following [CW2], we set, for $\rho>0, \Omega \equiv\left\{x \in \mathbf{R}^{d}: M f(x)>\rho\right\}$. By [FS1],

$$
V(\Omega) \leq \frac{c}{\rho} \int|f| M V d x,
$$

and therefore the theorem will follow from

$$
V\left\{x \notin \Omega: g_{\lambda}^{*}(f)>\rho\right\} \leq \frac{c}{\rho} \int|f| M V d x
$$

and interpolation with the $L^{2}$ inequality. 
Write $\Omega=\bigcup Q_{j}$, where the $Q_{j}$ are Whitney cubes, and set

$$
g(x) \equiv\left\{\begin{array} { l l } 
{ f ( x ) , } & { x \notin \Omega , } \\
{ f _ { Q _ { j } } , } & { x \in Q _ { j } , }
\end{array} \quad b _ { j } ( x ) \equiv \left\{\begin{array}{ll}
f(x)-f_{Q_{j}}, & x \in Q_{j} \\
0, & x \notin Q_{j}
\end{array}\right.\right.
$$

It is enough (see [CW2]) to show that

$$
V\left\{x \notin \Omega: g_{\lambda}^{*}\left(\sum b_{j}\right)>\rho\right\} \leq \frac{c}{\rho} \int|f| M V d x .
$$

Since $g_{\lambda}^{*}$ is subadditive, (42) will follow from

$$
\sum_{j} \int_{\mathbf{R}^{d} \backslash \Omega} g_{\lambda}^{*}\left(b_{j}\right) V d x \leq C \int|f| M V d x,
$$

which will in turn follow from

$$
\int_{\mathbf{R}^{d} \backslash \Omega} g_{\lambda}^{*}\left(b_{j}\right) V d x \leq C \int_{Q_{j}}|f| M V d x
$$

for some $C$ independent of $j$ and $\rho$. We shall now prove (43).

Let $x_{j} \equiv$ the center of $Q_{j}$. We need to estimate $b_{j} * \phi_{y}(t)$. We have two cases: (i) $y \leq l\left(Q_{j}\right)$; (ii) $y>l\left(Q_{j}\right)$. In case (i), the best we can do is $\left|b_{j} * \phi_{y}(t)\right| \leq C\left\|b_{j}\right\|_{1} y^{-d}$. In case (ii), we can use the fact that $\int b_{j}=0$ and get $\left|b_{j} * \phi_{y}(t)\right| \leq C\left\|b_{j}\right\|_{1} l\left(Q_{j}\right) y^{-d-1}$.

If $x \notin \Omega$, then $\left|x-x_{j}\right| \geq \operatorname{cl}\left(Q_{j}\right)$. Also, $b_{j} * \phi_{y}(t)=0$ unless $\left|t-x_{j}\right| \leq$ $c\left[y+l\left(Q_{j}\right)\right]$.

Thus, for $x \notin \Omega$, we have

$$
\begin{aligned}
& {\left[\int_{y \leq l\left(Q_{j}\right)}\left|b_{j} * \phi_{y}(t)\right|^{2}\left(\frac{y}{y+|x-t|}\right)^{d \lambda} \frac{d t d y}{y^{d+1}}\right]^{1 / 2}} \\
& \quad \leq C \frac{\left\|b_{j}\right\|_{1} l\left(Q_{j}\right)^{d / 2}}{\left|x-x_{j}\right|^{d \lambda / 2}}\left[\int_{y \leq l\left(Q_{j}\right)} y^{d \lambda-2 d-d-1} d y\right]^{1 / 2} \\
& \quad \leq C \frac{\left\|b_{j}\right\|_{1} l\left(Q_{j}\right)^{d / 2}}{\left|x-x_{j}\right|^{d \lambda / 2}} l\left(Q_{j}\right)^{(d \lambda-3 d) / 2} \\
& \quad \leq C \frac{\left\|b_{j}\right\|_{1} l\left(Q_{j}\right)^{-d}}{\left(1+\left|x-x_{j}\right| / l\left(Q_{j}\right)\right)^{d \lambda / 2}} \\
& \leq C\left|f \chi_{Q_{j}}\right| * \tau_{l\left(Q_{j}\right)}(x)
\end{aligned}
$$

where $\tau(x) \equiv 1 /(1+|x|)^{d \lambda / 2} \in L^{1}$, and inequality (44) is because $\lambda>3$. 
For the next part of the integral we consider two cases: $d=1$ and $d>1$. If $d=1$,

$$
\begin{aligned}
& {\left[\int_{l\left(Q_{j}\right)<y \leq\left|x-x_{j}\right|} \ldots \frac{d t d y}{y^{d+1}}\right]^{1 / 2}} \\
& \quad \leq C \frac{\left\|b_{j}\right\|_{1} l\left(Q_{j}\right)}{\left|x-x_{j}\right|^{\lambda / 2}}\left[\int_{l\left(Q_{j}\right)<y \leq\left|x-x_{j}\right|} y^{-4} y y^{\lambda} y^{-2} d y\right]^{1 / 2} \\
& \quad \leq C \frac{\left\|b_{j}\right\|_{1} l\left(Q_{j}\right)}{\left|x-x_{j}\right|^{\lambda / 2}} \cdot l\left(Q_{j}\right)^{\lambda / 2-2} \\
& \quad \leq C \frac{\left\|b_{j}\right\|_{1} l\left(Q_{j}\right)^{-1}}{\left(1+\left|x-x_{j}\right| / l\left(Q_{j}\right)\right)^{\lambda / 2}} \\
& \quad \leq C\left|f \chi_{Q_{j}}\right| * \sigma_{l\left(Q_{j}\right)}(x),
\end{aligned}
$$

where $\sigma(x) \equiv 1 /(1+|x|)^{\lambda / 2}$, and (45) is because $\lambda<4$.

On the other hand, if $d>1$,

(46)

$$
\begin{aligned}
& {\left[\int_{l\left(Q_{j}\right)<y \leq\left|x-x_{j}\right|} \cdots \frac{d t d y}{y^{d+1}}\right]^{1 / 2}} \\
& \quad \leq C \frac{\left\|b_{j}\right\|_{1} l\left(Q_{j}\right)}{\left|x-x_{j}\right|^{d \lambda / 2}}\left[\int_{l\left(Q_{j}\right)<y \leq\left|x-x_{j}\right|} y^{-2 d-2} y^{d \lambda} y^{d} y^{-d-1} d y\right]^{1 / 2} \\
& \quad \leq C \frac{\left\|b_{j}\right\|_{1} l\left(Q_{j}\right)}{\left|x-x_{j}\right|^{d \lambda / 2}} \cdot\left|x-x_{j}\right|^{d \lambda / 2-d-1} \\
& \quad \leq C\left\|b_{j}\right\|_{1} \frac{l\left(Q_{j}\right)^{-d}}{\left(1+\left|x-x_{j}\right| / l\left(Q_{j}\right)\right)^{d+1}} \\
& \quad \leq C\left|f \chi_{Q_{j}}\right| * P_{l\left(Q_{j}\right)}(x),
\end{aligned}
$$

where $P(x) \equiv 1 /(1+|x|)^{d+1}$ and (46) is because $\lambda>3 \geq 2+2 / d$.

Finally (since $\left.y /\left(y+\left|x-x_{j}\right|\right) \leq 1\right)$,

$$
\begin{aligned}
{\left[\int_{y>\left|x-x_{j}\right|} \ldots \frac{d t d y}{y^{d+1}}\right]^{1 / 2} } & \leq C\left\|b_{j}\right\|_{1} l\left(Q_{j}\right)\left[\int_{y>\left|x-x_{j}\right|} y^{-2 d-3} d y\right]^{1 / 2} \\
& \leq C \mid f \chi_{Q_{j} \mid * P_{l\left(Q_{j}\right)}(x) .}
\end{aligned}
$$



thus:

Therefore, if $x \notin \Omega$, and $d>1$, then $g_{\lambda}^{*}\left(b_{j}\right) \leq C\left|f \chi_{Q_{j}}\right| * P_{l\left(Q_{j}\right)}(x)$. And

$$
\begin{aligned}
\int_{\mathbf{R}^{2} \backslash \Omega} g_{\lambda}^{*}\left(b_{j}\right) V d x & \leq C \int_{\mathbf{R}^{d} \backslash \Omega}\left|f \chi_{Q_{j}}\right| * P_{l\left(Q_{j}\right)} V d x \\
& =C \int_{Q_{j}}|f|\left(P_{l\left(Q_{j}\right)} * V\right) d x \\
& \leq C \int_{Q_{j}}|f| M V d x,
\end{aligned}
$$

where (47) is because $P$ is even. If $d=1$ we get the same thing, but with $P$ replaced by $\sigma$. Q.E.D.

\section{REFERENCES}

[CWW] S. Y. A. Chang, J. M. Wilson and T. H. Wolff, Some weighted norm inequalities concerning the Schrödinger operators, Comment. Math. Helv. 60 (1985), 217-246.

[CW1] S. Chanillo and R. L. Weeden, $L^{p}$ estimates for fractional integrals and Sobolev inequalities, with applications to Schrödinger operators, preprint (1985).

[CW2] __ Some weighted norm inequalities for the area integral, Indiana Univ. Math. J. 36 (1987), 277-294.

[F] C. L. Fefferman, The uncertainty principle, Bull. Amer. Math. Soc. (N.S.) 9 (1983), 129-206.

[FS1] C. L. Fefferman and E. M. Stein, Some maximal inequalities, Amer. J. Math. 92 (1971), 107-115.

[FS2] _ , $H^{p}$ spaces of several variables, Acta Math. 129 (1972), 137-193.

[RF] R. Fefferman, Harmonic analysis on product spaces, Ann. of Math. 126 (1987), 109-130.

[G] J. B. Garnett, Bounded analytic functions, Academic Press, New York, 1981.

[GJ] J. B. Garnett and P. W. Jones, The distance in BMO to $L^{\infty}$, Ann. of Math. 108 (1978), 373-393.

[KS] R. Kerman and E. T. Sawyer, Weighted norm inequalities for potentials with applications to Schrödinger operators, Fourier transforms and Carleson measures, preprint (1984).

[K] D. Krutz, Littlewood-Paley and multiplier theorems on weighted $L^{p}$ spaces, Trans. Amer. Math. Soc. 259 (1980), 235-254.

[M] B. Muckenhoupt, Weighted norm inequalities for classical operators, Proc. Sympos. Pure Math., vol. 35, Amer. Math. Soc., Providence, R.I., 1979, pp. 69-84.

[Sch] M. Schechter, The spectrum of the Schrödinger operator, preprint (1987).

[St] E. M. Stein, Singular integrals and differentiability properties of functions, Princeton Univ. Press, Princeton N.J., 1970.

[U] A. Uchiyama, The Fefferman-Stein decomposition of smooth functions and its applications to $H^{p}\left(\mathbf{R}^{n}\right)$, Pacific J. Math. 115 (1984), 217-255.

[W1] J. M. Wilson, Weighted inequalities for the dyadic square function without dyadic $A_{\infty}$, Duke Math. J. 55 (1987), 19-49.

[W2] _ _ A sharp inequality for the square function, Duke Math. J. 55 (1987), 879-887.

[W3] _., $L^{p}$ weighted norm inequalities for the square function, $0<p<2$, Illinois J. Math. (to appear).

Department of Mathematics, University of Vermont, Burlington, Vermont 05405 